\title{
Suitability of Tilting Technology to the Tyne and Wear Metro System
}

\author{
Agajere Ovuezirie Darlton $^{2} \cdot$ Marin Marinov ${ }^{1}$
}

Received: 14 December 2014/Revised: 27 February 2015/Accepted: 6 March 2015/Published online: 28 May 2015

(C) The Author(s) 2015. This article is published with open access at Springerlink.com

\begin{abstract}
This paper attempts to determine the suitability of tilting technology as applied to metro systems, taking the Tyne and Wear Metro as its base case study. This is done through designing and implementing of several tests which show the current metro situation and reveals possible impacts on ride comfort and speed, in case tilting technology has been implemented. The paper provides brief background literature review on tilting technology, its different designs and types, control systems, customer satisfaction and history on the Tyne and Wear metro system. Ride comfort evaluation methods, testing of the Metro fleet comfort levels and simulation modelling through the use of OpenTrack simulator software are also introduced. Results and findings include test accuracy and validations and suggest that although tilting technology could be beneficial with respect to speed (minimal improvements) and comfort, implementing it to the Tyne and Wear metro would be an unwise decision owing to the immense amount of upgrades that would be needed on both the network and the metro car fleet. Therefore, recommendations are subsequently made on alternative systems which could achieve or surpass the levels of comfort achievable by tilting technology without the need for an outright overhaul of lines and trains.
\end{abstract}

Marin Marinov

marin.marinov@ncl.ac.uk

1 NewRail, Mechanical and Systems Engineering School, Newcastle University, 2nd Floor, Stephenson Building, Newcastle upon Tyne NE1 7RU, UK

2 Mechanical and Systems Engineering School, Newcastle University, Stephenson Building,

Newcastle upon Tyne NE1 7RU, UK

Editor: Baoming Han
Keywords Tilting technology - Tyne and wear metro · OpenTrack · Simulation - Lateral acceleration · Ride comfort

\section{Introduction}

Tilting trains, currently gaining popularity in the railway market has been a concept considered ever since the late 1930s. Individuals have pondered on ways of improving rail speeds without compromising passenger ride comfort and possible derailment of the train vehicles. Previously, trains remained limited in speed by the curvature of rail tracks. Trains generally need to slow down to an acceptable speed in other to successfully negotiate curves. A fitting example of disasters caused by over-speeding in curves is the 2013 Spanish high-speed rail disaster [1] where the train derailed due to over-speeding. Despite these incidents, there are still paramount demands on railways to deliver high-speed transportation for their passengers in order to compete effectively with other modes of transportation. A tilting train refers to a train capable of negotiating a bend or curve at speeds greater than that limited by the curve through the use of active or passive tilting mechanisms. When a bicycle or motorcycle negotiates a curve at high speeds, the driver or rider tilts the vehicle at an angle that is a function of horizontal speed and radius of curvature of the curve thus reducing the magnitude of lateral acceleration on the vehicle. This behaviour is also seen in speeding bodies from animals, track athletes, ice skaters and many more. Automobile and rail vehicles cannot mimic the tilts achievable by two wheel vehicles most specifically because they (automobiles and rail vehicles) are four or more wheel vehicles. In the automotive industry for example, attempts have been made to increase speeds around curves through the use of canted roads. Canted 
roads can most commonly be seen in the racing industry and most commonly in a NASCAR High-speed track. The railway industry can also use cants, but the angles achievable are relatively small and do not necessarily meet with growing high-speed demands. In the automobile industry, road cants or super-elevations can reach angles of $30^{\circ}$ or more whilst in railway, cant levels only reach about $3^{\circ}$. Tilting trains use their mechanisms in increasing the angle of tilt hence increasing the amount of speed the train can use in negotiating the curve. Tilting trains are seen as a cost-effective way to meet passenger demands without spending valuable funds on reconstruction of high-speed straight line tracks. The design of a tilting train is shown below. A component called the tilting bolster is mounted on the bogie and the car body is in turn mounted on the bolster. The bolster is connected to swing links which are in turn connected to an actuator. Actuators may be in the form of a hydraulic cylinder using hydraulic oil as its working fluid, an air cylinder using compressed air as its working fluid or an electromechanical actuator. Controls, sensors and programmes are put in place to ensure smooth transitions of the train as it approaches, negotiates and exits the curve. Apart from the benefits of high speed, improved ride comfort is also achieved as the extra cylinders and air springs act as secondary dampers reducing the amount of vibration transmitted from the track, through the bogies to the passengers.

\subsection{Motivation}

High-speed rail history in the UK suggests that when considering the costs involved in building new rail tracks for the purpose of increased rail speed, tilting technology would be a worthwhile solution. Tilting trains enable increased speed on regular tracks however there are not many tilting trains in service today, especially in the UK. As it turns out, increased speed is not the only benefit of tilting vehicles. Ride comfort and quality can also be significantly increased. Over the next 15 years, the Tyne and Wear metro intends to replace current Metro vehicles with new rolling stock. Would tilting technology be beneficial? Would customer satisfaction be increased through increase in ride quality and/or increased speed (reduced commuting time)? Could tilting technology be applied to intercity trains not necessarily because of increased speed, but increased comfort? These questions pose significant motivation for possible study so as to conclusively understand and determine sufficient and satisfactory answers.

\subsection{Aims and Objectives}

The objective of this paper is to determine and analyse the suitability of tilting technology to the Tyne and Wear metro. Previous works on tilting technology have mainly been implemented on high-speed rail systems and hardly any research has been done to implement it on intra-city metro systems despite a growing demand for higher comfort and speed. This project attempts to determine the possible impact of tilting on metro systems in terms of speed, ride comfort, motion sickness and also determine if tilting can and should only be useful in the High-speed rail sector. It is worth to note that metro systems hardly travel at the required high speeds which would necessitate the use of tilting technology, however this project will attempt to identify the current levels of comfort in the current metro and also determine how beneficial tilting technology could be to ride comfort and subsequently overall customer satisfaction.

\subsection{Research Methodology}

This project first determines the current level of customer comfort in the metro by showing the correlation between commuting speed and vibration levels whilst making comparisons with scales determined according to ISO 2631:1997. Track speed and vibration measurements were measured using accelerometer sensors located in a Samsung galaxy grand duos smart phone where smart phone apps, vibration monitoring and speedview 2.31 are used to record these data and return values in graphical format. Comparisons of collected data are then made with speed simulation data from OpenTrack to determine the differences in performance of the train vehicle and correlations made with vibration data thus showing how much of an improvement could be made through the implementation of better ride comfort and speed.

\section{Literature Study}

A tilting train refers to a train that has a tilting mechanism which enables it to travel at higher speeds compared to non-tilting or conventional trains. Nam-Po Kim and TaeWon define tilting trains as trains capable of increasing curving speeds without reducing the safety or comfort of passengers [2]. With growing competition from other modes of transport, railway industries have recognised and acted on the need of increasing travel speeds thus reducing travel time on passengers. Another relevant reason for tilting trains stems from the need for better comfort for passengers. Tilting trains achieve these demands excellently without the need of reconstruction of regular lines in order to lay new straight line tracks. Over the years, over 5000 tilting vehicles have been produced worldwide by different suppliers [3].

Despite the many benefits of tilting technology, it still has its drawbacks. The most relevant being motion 
sickness. Passengers have reported cases of nausea, dizziness and vomiting in severe cases. Motion sickness is not necessarily uncommon in the transportation industries. Non-tilting trains, although not as much as tilting trains, have reported passengers experiencing motion sickness [4]. There is currently a general understanding that having tilting trains which compensate for $100 \%$ of lateral acceleration poses high risks of inducing motion sickness [4]. The Swedish preferred the tilt compensation method to compensate for $65-70 \%$ of lateral acceleration on curves [4] as is currently used in the Swedish X2000 tilting train. Several methods have been employed to find out the underlying cause of motion sickness, and research has shown that passengers complain the most when the train uses a passively tilted system. Actively tilting train does record motion sickness cases however the numbers coincide closely with non-tilting trains [4].

There are several types of tilting technologies currently being used or developed in the railway industry. The Italian pendolino developed by FIAT [5] stands out as the most popular and is being used today in the United Kingdom West Coast mainline. The Swedish X2000 train series developed by ASEA Brown Boveri [5]. Other developed or currently being developed technologies include the long air spring system developed in Japan [6] and the hybrid system also developed in Japan [7].

\subsection{Tilting Technology and Mechanisms}

The concept of tilting technology lies in the physics of centrifugal and centripetal forces. When a vehicle travels around a curve, bodies in the vehicle experience forces which tend to push them in a direction towards the outside of the curve. This force is called centrifugal force. A more elegant definition states that "Centrifugal force is an apparent force which draws a rotating body away from the centre of rotation and it is caused by the inertia of the body as the body's path is continually redirected". It is important to note that centrifugal force is an apparent force. That is a force introduced into a system in order for Newton's laws of physics to be satisfied. The picture below shows a schematic example of how this force is experienced. In an automobile vehicle, bodies in the vehicle experience this force. If the vehicle goes too fast around the curve, there is a high potential of the vehicle turning over and potentially causing fatal injuries. It is for this reason that vehicles slow down before the beginning of a curve so as to limit the magnitude of this force. This same concept also applies to train vehicles.

Tilting technology enables the trains to tilt at an angle towards the centre of the curve which reduces the magnitude of the centrifugal force experienced by passengers enabling the train to travel at a higher speed whilst improving or maintaining the amount of comfort for the passengers. A schematic diagram of the forces involved is given below. The chosen train vehicle is the Italian pendolino train vehicle with its pendulum system. In actuality, it does not matter which sort of system is chosen as the experienced forces do not change

$$
\begin{aligned}
& \text { If } \theta_{\mathrm{v}}=\text { Car body tilt angle } \\
& \theta_{0}=\text { Track cant angle } \\
& \text { and } \ddot{Y}=\text { Lateral acceleration (centrifugal acceleration) } \\
& \ddot{Y}=\frac{V^{2}}{R} \cos \left(\varphi_{\mathrm{c}}+\varphi_{\mathrm{t}}\right)-g \times\left(\varphi_{\mathrm{c}}+\varphi_{\mathrm{t}}\right),
\end{aligned}
$$

where $V$ is the velocity, $R$ is the radius and $g$ is the constant of gravity.

As shown in the Fig. 1 above, one can see that the amount of lateral acceleration or centrifugal force experienced by the passengers depends on the summation of both centrifugal and centripetal forces. Tilting technology increases the amount of centripetal force on the vehicle by shifting the centre of gravity of the car body inwards of the curve and this is given by the formula $g \sin \left(\varphi_{\mathrm{c}}+\varphi_{\mathrm{t}}\right)$. Centrifugal force given as $\frac{V^{2}}{R} \cos \left(\varphi_{\mathrm{c}}+\varphi_{\mathrm{t}}\right)$ is a function of the speed of the car body, radius of the curve and total angle of tilt. As speed increases, centrifugal force increases significantly. A reduction in the resultant centrifugal force results in better ride comfort for the passengers and higher possible speeds for the train vehicle.

\subsubsection{Tilting Technology Types}

There are two types of tilting mechanisms

1. Passively tilted trains

2. Actively tilted trains

Passively Tilted Trains Also called naturally tilted trains, these are trains which rely on the physical laws of physics without any actuators, control systems or power sources initiating tilt. The tilt centre of the train is purposely located above the centre of gravity and this enables the train to tilt when negotiating curves due to its weight [9]. Should the tilt centre of the train be located at the same point or below the vehicle centre of gravity, the system would become highly unstable with passengers experiencing high levels of discomfort. According to [3], damping of the tilt motion is required to control the otherwise relatively undamped car body roll motions. This is because if left undamped, the car body would end up reacting in an unconventional manner to the slightest of curves which can be very uncomfortable for the passengers.

Several examples of systems with passive or natural tilting includes 
Fig. 1 Pendulum tilting system. a Tilting train with tilting bolster, $\mathbf{b}$ basic concept of tilting [8]

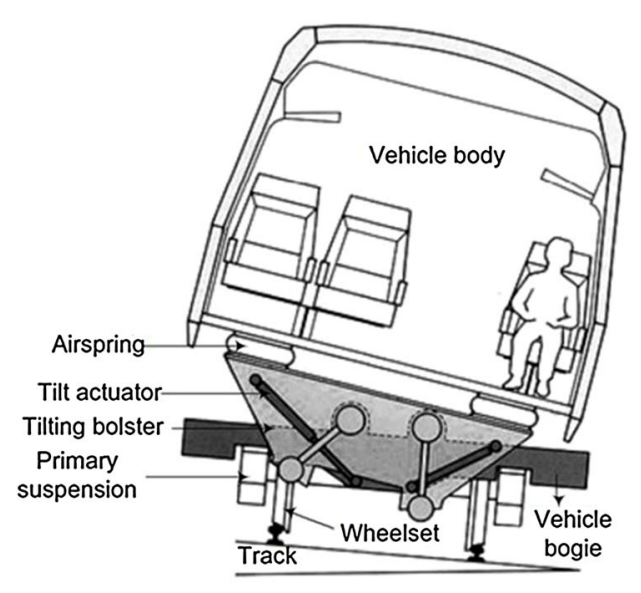

(a)

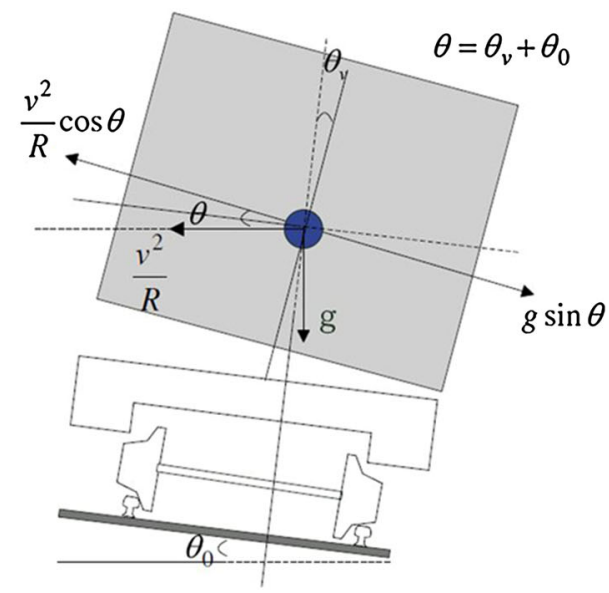

(b)
1. Swing bolster with circular arc guide

2. High-positioned air spring

3. Inclined anti-roll bar links

Swing Bolster with Circular Arc Guide This is a natural tilting solution used in early Japanese tilting trains. In this system, the car body sits on air springs, which is mounted on a swing bolster (tilting bolster). The tilting bolster in turn sits on rollers or bearings which allow motion along a circular path. According to Persson [3], the car body's centre of gravity is located about $600-900 \mathrm{~mm}$ lower than the location of the tilt centre. There are two major tilting components in this system. The first is the tilting bolster and the second is the roller bearings which the bolster sits on. This system allows a $5^{\circ}-6^{\circ}$ tilt capability of the car body. The system uses roll dampers which are installed between the bogie frame and tilting bolster to limit overshooting [3].

High-Positioned Air Spring In this system, centrifugal acceleration forces the car body to tilt around the centre of a pair of air springs which are installed on a pair of pillars which sit on the train bogie. The air springs also acts as a secondary suspension system giving more stability to the car body and this arrangement is mostly used in Talgo systems [3]. Roll stiffness is controlled by an electro-pneumatic valve which connects both air springs. This system has a simple structure and it realises natural tilting without the implementation of complex devices or mechanisms.

Inclined Anti-roll Bar Links In the high-positioned air spring system, the air springs are located closer to the top of the car body. In this system, the air springs are installed below the car body. This system has natural tilting capabilities but can be better considered as a normal train system with better compensation for suspension flexibility. Generally it can achieve a tilt angle of about $2^{\circ}$.

Advantages of passively tilted trains
1. The system is relatively simple to implement.

2. System simplicity enables low initial and maintenance costs.

3. Hardly any control system required.

Disadvantages of passively tilted trains

1. System has higher potential to induce motion sickness in passengers.

2. Safety issues like overturning arise as a result of lateral shift of the car body's centre of gravity.

This system is being phased out as it poses safety concerns due to the lateral shift of the centre of gravity of the car body.

Actively Tilted Trains Actively tilted trains rely on active technology controlled by sensors and programmed electronics. Tilt is executed by actuators which may be hydraulic or electromechanical in nature. Also the centre of gravity does not change its position thus it is considered a much safer alternative to the passive tilt system [10]. Control systems are installed in order to initiate tilt in a timely fashion so as to efficiently utilise the vehicle capabilities. Most especially in natural tilting systems where there are no controls, a train vehicle may begin to tilt too late and this can be highly uncomfortable for passengers and potentially catastrophic to infrastructure. Control systems will be covered in detail in the next section.

Identified examples of systems with active tilting mechanisms include

1. The Japanese pneumatic system

2. Hybrid tilting system

3. Italian pendulum tilting system

The Japanese Pneumatic Mechanism This system developed in Japan comprises a simple air cylinder used as an actuator to initiate tilt. The car body sits on a pair of air 


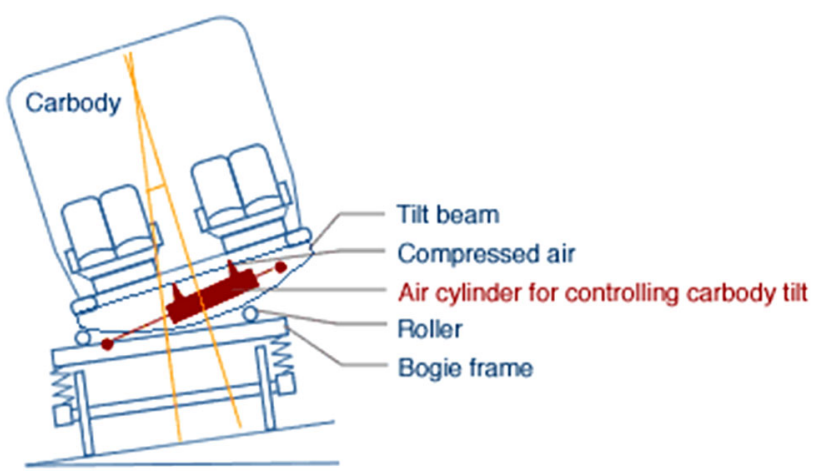

Fig. 2 Schematic illustration of Japanese tilting mechanism [11]

springs which act as dampers and they in turn sit on the tilting bolster. The bolster is mounted on rollers which allow the body to roll into its desired tilting position as shown in Fig. 2. The system shares the same power source with the pneumatic brake system. It is a compact (space saving) system, which is preferable for limited under-floor mounting space of the rolling stocks used on narrow gauged tracks. Without using hydraulic power, the system can be easily cleaned, maintained and generally have low cost of implementation. Also the simplicity of the system contributes to the element for speed up and assures less pressure or damage to the tracks. This system has its merits but scores low in terms of vibration and passenger comfort. A critical look at the schematic diagram would show that the tilting bolster sits on bearings for smooth tilting. However, this design would mean that the bogie would have a high value of vibration transmissibility compared to other tilting systems.

Hybrid Tilting System The hybrid tilt system (Fig. 3), developed by a research team uses a combination of two conventional tilting systems in order to gain extended tilt angles of up to $8^{\circ}$. The first system uses a conventional hydraulic actuator capable of initiating a tilt angle of $6^{\circ}$. The second uses the secondary air spring suspension system to initiate an additional tilt angle of $2^{\circ}$. The combined system would enable a tilting train achieve a speed of
$+50 \mathrm{kmph}$ as total tilt angle comes to $8^{\circ}$ [7]. They also claim that the system would be safe and comfortable for passengers as feedback and preventive control systems would be put in place to ensure synchronisation of both tilting systems. Considering that this project aims to implement tilting systems in light rail or metro systems, this tilting mechanism will not be considered. A critical observation indicates that the system is still in its research phase and proper feasibility studies have yet to be undertaken. Motion sickness would be an issue and when one considers a vehicle with tilt capability of $6^{\circ}$ only, it becomes slightly confusing why a need for an additional $2^{\circ}$ would be needed to go faster.

Italian Pendulum Tilting System The Italian pendolino (Italian for pendulum) trains are quite common and is also currently used in the UK. This system shown in Fig. 4 is an active controlled system using hydraulic actuators which connects to swing links which are in turn connected to the tilting bogie. The system is safe, reliable and although slightly more complex than other tilting systems, it does have a higher positive feedback in terms of passenger comfort and motion sickness. This system would be a good choice to consider when considering implementation on the current metro system. Reason for this is its proven reliability and its already widespread patronage.

\subsection{Control Systems}

In tilting trains, naturally tilting systems hardly need any controls to initiate tilt. However, most naturally tilting systems can be upgraded with an actuator in order to improve their performance and give more control to operators and/or computer systems. Such are most actively tilting systems. As defined previously, actively tilting trains require some sort of device or component in other to initiate tilting.

Several control system types have been developed over the years with each having its merits and demerits. The three prominent control systems are

Fig. 3 Hybrid tilting bogie [7]

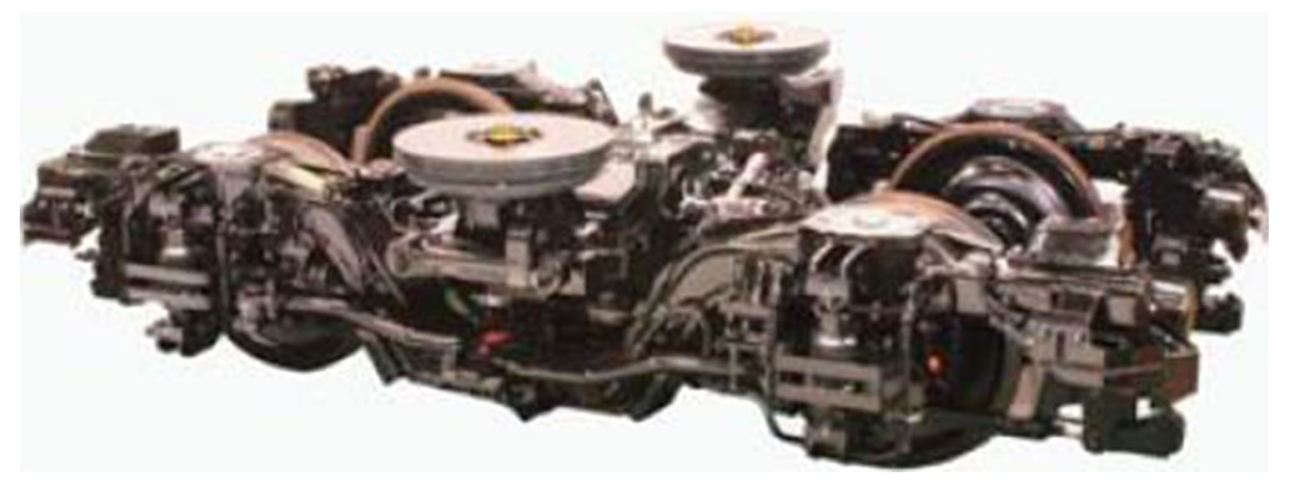


Fig. 4 Pendulum tilt system [12]

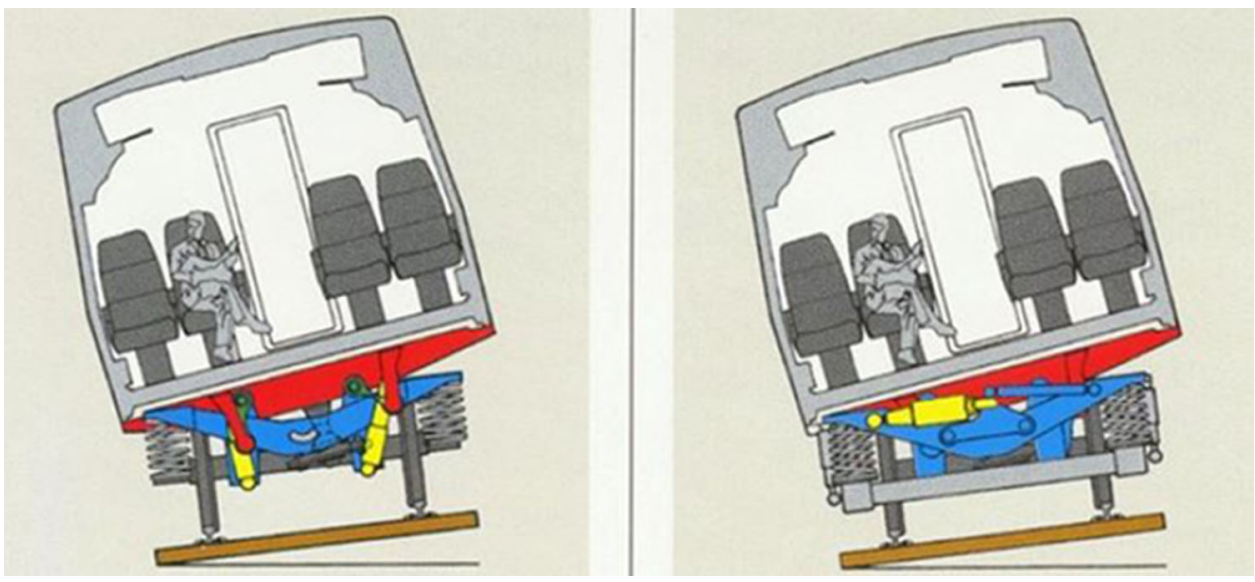

1. The body feedback control system

2. The bolster feedback control system

3. Reference transducer control system

The body feedback systems were first developed and worked by installation of an accelerometer in the body of the car which reads the total lateral force on the car body. The body accelerometer then sends a signal to the controller which then compensates with tilting at the actuator. This system had to be upgraded due to numerous stability problems arising due to low frequency movements in the secondary suspension. The bolster feedback system used accelerometers installed in the tilting bolster of the bogie. Although this system was much more efficient than the previous version, it still had to be upgraded. The main reason was because the tilting train when approaching a curve could be overly compensated or under compensated. The reference transducer system where a transducer is installed on the bogie receives signals from another transducer installed on the tracks. The signals include information of the curve, permissible speed and required tilt all of which are important factors which leads to an efficient negotiation of the curve. The reference transducer system is often called predictive tilt control system. Rightly so it ensures the train control systems possess the necessary information before it reaches the curve. Having this information beforehand, the system has proven to be a more stable and enjoyable system. It has also been found to have a positive impact on motion sickness which turns out to be the most challenging factor in terms of passenger comfort.

Figure 5 is a schematic diagram which shows how control systems have evolved over time and it is easily obvious why the reference transducer system is considered more efficient than the other systems. An example of tilting train systems which use the reference transducer control system is the Japanese tilting trains. These trains use wayside information to improve the performance of tilt. These systems combine an automatic train protection

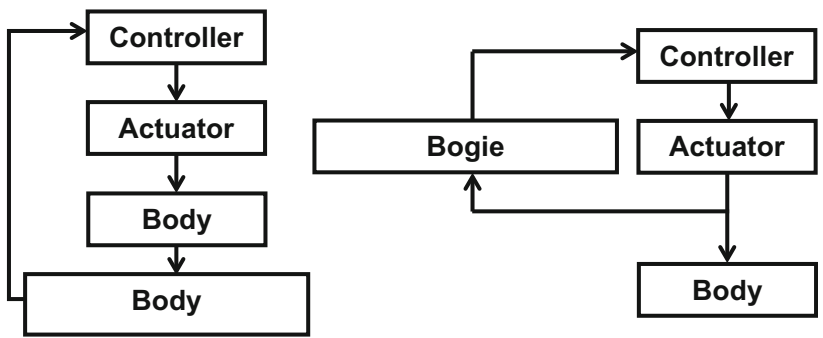

$\begin{array}{ll}\text { Body Feedback system Bolster Feedback } & \end{array}$

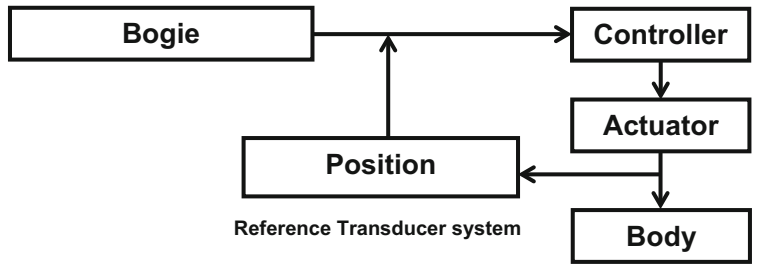

Fig. 5 Layout of Tilt control systems modified from [10]

(ATP) system, where track information is sent from a wayside element to the ATP system in the train and this is then transmitted to the tilt controller. There are debates on whether system track data should be sent to the train via wayside elements or if the data can be stored beforehand in the train [13]. Experts have debated on which is the best with the former having a higher vote of confidence. The reason why is because should there be any track changes, it could be easily registered unlike the latter system which could pose potential safety concerns if there are any track changes like a damaged track. However, there is a possible flaw in terms of cost to the wayside system. If there are sensors and transducers that have to be put in place before every track curve, it may become more expensive to enact compared to if there is a single system located in the train where all track data are stored. It may turn out that the system which is best may differ in pertaining to different train systems and their requirements. A compromise 
Fig. 6 Illustration of predictive tilt control system [15]
Concept of Controlled Tilt System

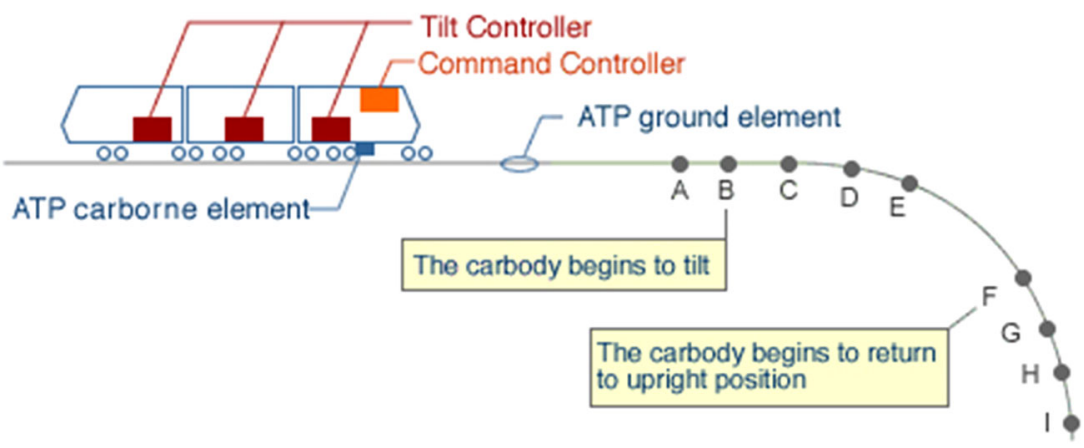

between system requirements and cost may ultimately lead to the best decision. There have also been claims that the Spanish supplier Construcciones y Auxiliar de Ferrocarriles (CAF) has in the series R-598 showing that a system based on stored track data can work without wayside input [14]. According to the claim, the stored track data can either be based on a track data register or on a train measured track data (Fig. 6). A positioning system would be required to pick the right data at the right time.

Important design parameters for a control system according to [14] include

- Filtration of input signals and optimization of response time delays

- Good tilt compensation ratios

- Designing control loops to minimise undesired car body behaviours ultimately optimising fast response.

Frostberg [12] went further to state that "control loops have to be designed together with the car body vehicle suspension dynamics so as to minimise any interference with the tilt system".

\subsection{Tyne and Wear (Newcastle) Metro}

The Tyne and Wear metro is a light rail service system located in the north east of the UK. It serves over 60 stations spanning the majority of North East city centers all the way from Newcastle to Sunderland. Having been in operation since 1980, several refurbishments and overhaul of the train fleet has been done over the years. However, with the fleet currently over 30 years old, and set to remain in service for the remainder of the next decade, there is a growing need for modernization.

Table 1 shows the specifications of the metro car being used in on the Tyne and Wear network. Power is obtained from $1500 \mathrm{~V}$ overhead lines through a pantograph which can be designed in a manner so as not to be affected during tilting, a weight of about 39 tonnes when fully laden and it currently seats 68 people sitting and 232 standing. The
Table 1 Metro car specifications

\begin{tabular}{ll}
\hline Construction & $1974-1984$ \\
\hline Beginning of service & 1980 \\
Train set formation & 2 cars \\
Train set capacity & $68 \mathrm{sitting}, 232$ standing \\
Maximum speed & $50 \mathrm{mph}(80 \mathrm{~km} / \mathrm{h})$ \\
Weight & 39 metric tonnes \\
Power system & $1500 \mathrm{~V} \mathrm{DC}$ overhead lines \\
Current collection method & Pantograph \\
Track gauge & $1435 \mathrm{~mm}(4 \mathrm{ft} 81 / 2 \mathrm{in})$ \\
\hline
\end{tabular}

train has the capabilities of reaching a maximum speed of $80 \mathrm{~km} / \mathrm{h}$.

\subsubsection{Rolling Stock}

The rolling stock of the Tyne and Wear has remained unchanged ever since beginning of operations, which puts them at over 30 years old. With several refurbishment schemes in place since 2010, the main goal is to extend their service life till the next 10-15 years after which they would be replaced by a new fleet set. The current fleet is made up of a total of 90 train sets in a two car per unit formation which can also be coupled together in pairs to form a four car unit formation. The metro also has in operation a three battery run electric locomotives constructed by Hunslet which are used to pull engineering trains around the network [17]. The metro train sets are each around $56 \mathrm{~m}$ long, using articulated six-axle twin vehicles, with 68 passenger seats and spacing for up to 200 standing passengers. All 90 of the metro fleet are in use the system; uses a power supply of $1500 \mathrm{~V}$ DC via overhead lines. A pantograph is used to transmit the current from the overhead lines to the train Fig. 7. The trains are also equipped with an air braking system and also an electro-magnetic emergency braking system. 


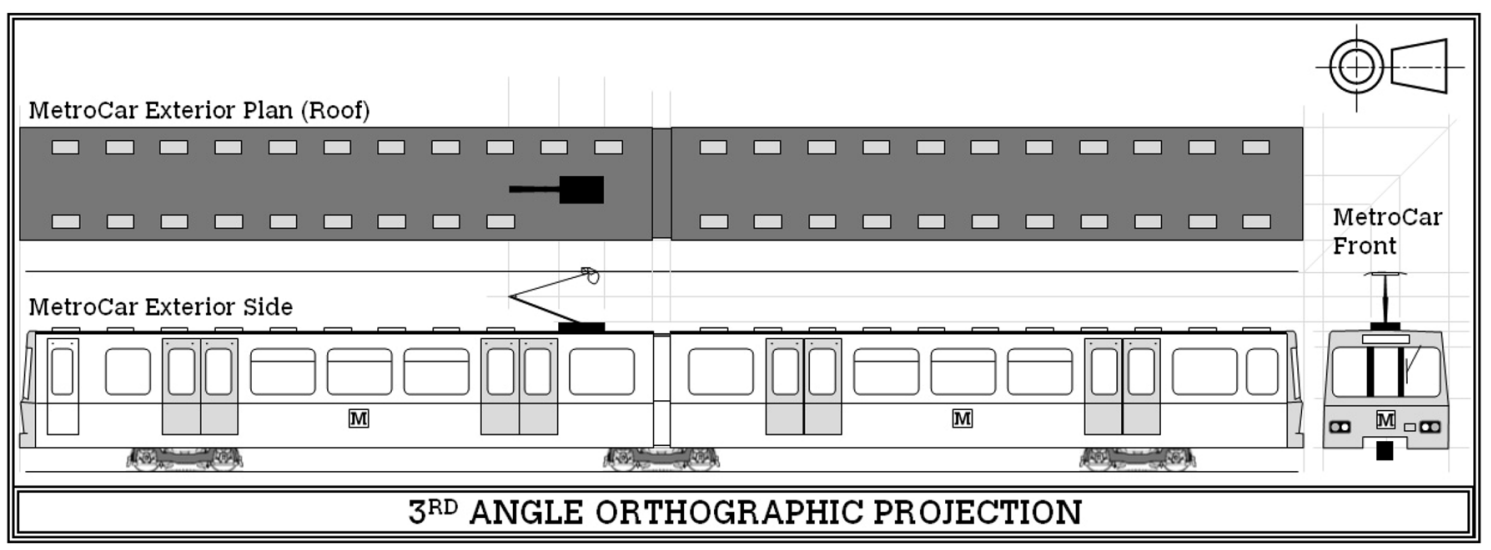

Fig. 7 Schematic Illustration of Tyne and Wear Metro Car [16]

Fig. 8 Current Tyne and Wear Metro Network [16]

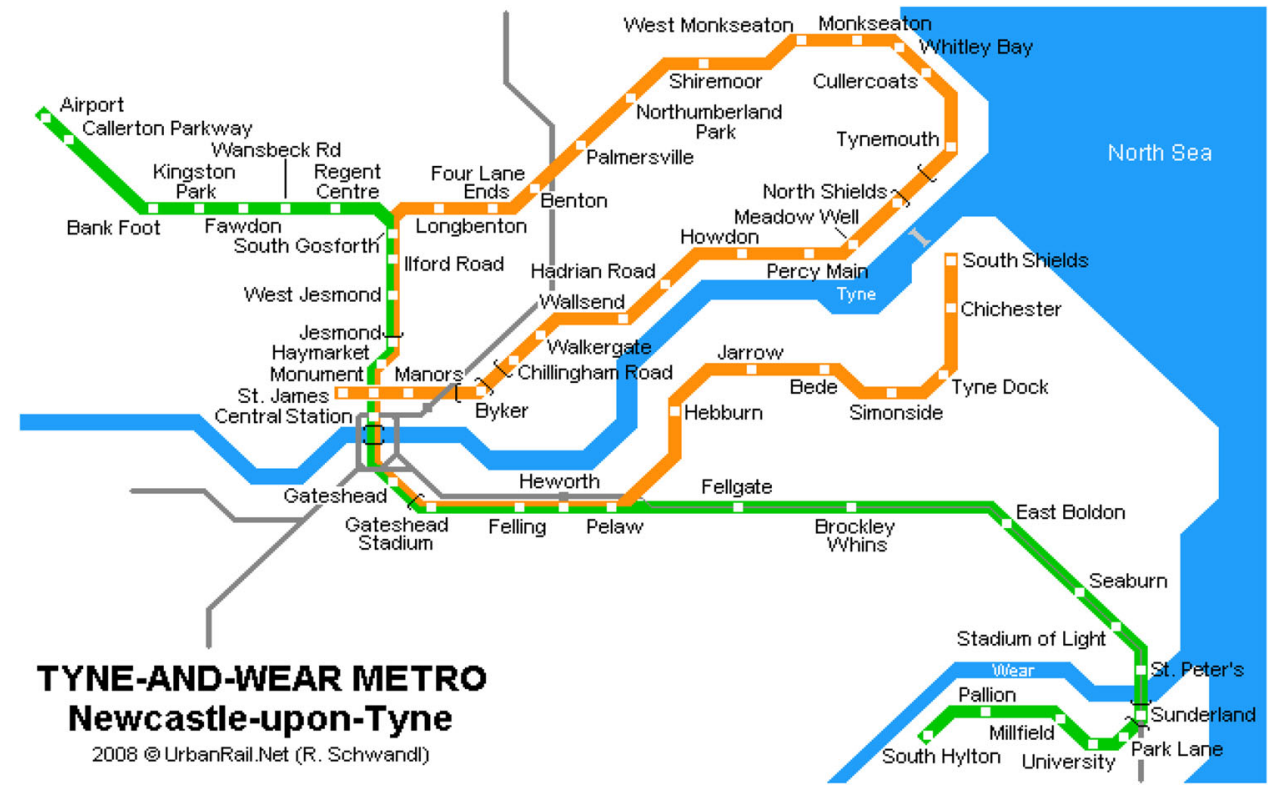

\subsubsection{The Tyne and Wear Network}

The Tyne and Wear network consists of two lines, called the green and yellow lines. As shown in Fig. 8, the green line begins at Newcastle Airport and runs through Newcastle City centre all the way to South Hylton in Sunderland. The yellow line begins at St James' Stadium, goes all the way to Four Lanes End and loops on itself before crossing the Tyne River heading to South Shields.

Figure 8 shows the Tyne and Wear Metro system including its green and yellow lines encompassing its 60 stations. It is easily obvious that the relative distances between most of the stations like Jesmond and Haymarket are quite small. However, one could also notice a much larger distance between some other stations like Pelaw and Hebburn or Brock whins and East Boldon. Since considerations are also going to be made for speed, it would be important to note the relative distances between each station and identify sections where the metro reaches its maximum speed. This would help identify possible sections where the metro may be capable of surpassing its maximum speed.

\subsubsection{Future Plans}

With the current metro fleet showing signs of old age as the come to a 35 year mark, plans have been put in place by Nexus for a full replacement by 2030. In a plan called the Metro strategy, Nexus outlines its future plans and how it would be making its case to the United Kingdom Government in hopes of the realisation of its visions and also the expansion of the current metro network including the possibilities of tram services in areas not covered by the metro. As shown in the network map below, several route expansions have been proposed. It is worth noting that the 
decisions for expansion would only be made after careful considerations of cost, investment benefits and also the general effect on the economy. In a survey conducted by Nexus, it was discovered that an investment of one pound in the metro yields a return of eight pounds [18]. This gives a sort of incentive on the values of the metro despite its being highly subsidised by the government. The network expansions being proposed include Silverlink in North Tyneside, Team Valley in Gateshead, The MetroCentre and Newcastle's West End [19].

\section{Customer Satisfaction, Ride Comfort and Motion Sickness}

Lauriks et al. [21] define comfort as "the wellbeing of a person or absence of mechanical disturbance in relation to the induced environment" [20]. It also stipulates that such wellbeing is achieved or disturbed based on a number of different factors which could be physiological in nature, for example, individual expectations and individual sensitivity and stress, or physical in nature like vehicle motions, noise, temperature and seating characteristics [20]. When looking at a situation combing some or all of these factors, the perception of comfort based on similar vibration values may be judged as uncomfortable in a given environment and comfortable in another environment [20]; it also defines ride quality as an entity representing a passenger's judgement of comfort level of a specific journey based on all experiences encompassing all factors. In every railway system, maintaining a healthy customer base is an essential part to every network's future. A sustained and/or increasing number of passengers ensure that the business grows which in turn provides avenues for service improvements, network extensions and ultimately increasing the growth of the immediate economy. The only way in which such strong, stable and sustained customer base can be achieved is through endeavours made to ensure that the overall customer satisfaction factors are made the highest priority. A satisfied customer is a happy customer; thus, if railway systems put measures in place to ensure customer satisfaction, then overall business will experience growth.

Over the years, several literatures have been studied in order to identify the factors which most influence customer satisfaction. As much as each and every factor remains absolutely important, some are consistently rated over time as being more important than others [21]. According to surveys conducted by different sources, identified customer satisfaction factors include

- Punctuality

- Reliability

- Station and train cleanliness
- Comfort level of train

- Train cleanliness

- Station condition

- Staff availability

- Passenger behaviour

- Train running information

- Safety and security

- Transport integration

With different authorities on the subject having different views on which of these factors have the most influence on overall satisfaction of passengers, The Transport for Greater Manchester amongst others claims that the key factors which would be the best predictors of overall passenger satisfaction in a train journey are

1. Cleanliness inside the trains

2. Comfort of the seating area(ride quality)

3. Punctuality and reliability

These findings coincide properly with other authorities like passenger-focus or Nexus thus making their findings increasingly accurate.

The current metro system scores very well on the subject of punctuality and reliability as there are hardly any breakdowns of the trains due to quality and preventive routine maintenance. The cleanliness of the train is also very good as the train vehicle is consistently cleaned before embarking on new journeys. However, the overall comfort of the train vehicle is not so good. Passengers frequently complain of vibrations felt during the journey and these can become concerning especially for passengers more susceptible to motion sickness. The current network of the metro covers a total distance of $77 \mathrm{~km}$ with average distances between stations around $2 \mathrm{~km}$. On a short journey, say from south Gosforth to central station, passengers may not feel at all bothered by the vibrations of the train vehicle. This could be because they might be more energetic and would not feel any impacts from the vibrations. However, if we look at a case of someone who has travelled a couple of hours by flight and has to use the metro from the airport to Fellgate, it becomes understandable that such a person would be impacted by the amount of vibrations experienced. During a personal survey, it was observed that taking a journey in the morning, after a full breakfast and ready to start the day, there was little thought as to the magnitude of vibrations felt in the train vehicle. However, when taking the journey back home at the end of the day, the magnitude of vibration was almost unbearable as the train could not arrive at the destination station soon enough. On subsequent days, the experience was the same. This leads to a conclusion that when it comes to passengers who have already had a long day, the comfort level of the current metro system scores very low. 


\subsection{Motion Sickness}

Motion sickness is said to occur when a person or group of people feel dizzy, nauseated or sick specifically due to conflicting body responses to real, perceived or anticipated movement. Mostly experienced by individuals in moving vehicles, for example ships, airplanes, automobiles or trains, it is caused when the human brain does not understand signals sent to it by the body's natural sensory organs (eyes, ears and more) which it makes to assume that the body may be host to foreign objects which may need to be expelled. This leads to several impulses where the individual feels like dizziness, vomiting, increased temperatures, increased salivation and many other symptoms. Reported cases of motion sickness date as far back as 5th century BC when Hippocrates stated that sailing on the sea creates disorder in the body [3]. Observations have shown that drivers hardly experience motion sickness leading to a general understanding that having forehand information significantly reduces the likelihood of experiencing these symptoms.

\subsubsection{Motion Sickness in Trains}

Tilting trains have been claimed to cause more motion sickness cases than non-tilting trains although these claims remain debatable. Reasons for debate range from knowledge about cases of motion sickness that are generally reported in both train types. In many surveys that have been made, a stark contrast in results show that possibly motion sickness may also be affected on an individual and geographical basis. In a survey done in 1964, it was reported that $0.13 \%$ out of 370,000 individuals suffered symptoms related to motion sickness on non-tilting trains in America, whilst on a similar test in Tokyo, $18 \%$ of passengers experienced motion sickness symptoms [3]. Conflicting test reports have also been noticed in reports by Frostberg on tests in Sweden (Swedish X2000 tilting train) and Norway where the Swedish report claimed that there was a clear correlation between tilt compensation and motion sickness, whilst the Norway report claimed that there was no significant correlation between tilt compensation and motion sickness [10, 22]. Persson [10] also claims that the extent of reported cases in Europe seems to be less than those reported in Japan. These reports tend to show a pattern of differences pointing to individual, geographical and psychological reasons. Also during a test done by this project, it was noticed that when taking a train ride in the morning, thoughts of being uncomfortable were virtually non-existent compared to taking a train at mid-day or worse, at the end of the day. It can be said that depending on many different factors including
- State of mind

- Individual susceptibility

- Sensitivity to motion

- Daily work stress

- Travel fatigue

- Geographical area

- Track conditions

- Train characteristics

- Track gauge

- Magnitude of tilt compensation

Some individuals could show symptoms of motion sickness in some cases or situations and not show any in other situations even when other people show these symptoms. A typical example is if an individual, who has been on a $6 \mathrm{~h}$ flight into Newcastle, takes a train ride and is exposed to several train movements, he/she may experience motion sickness compared to another individual who simply went to the airport as an escort or family member to pick them up. Table 2 shows the ranges of symptoms of motion sickness ranging from gastro-related issues to deeply psychological issues. Though not an exhaustive list, it can be seen that symptoms occur in line with individual states. An individual who may have had a heavy meal in the morning would be more likely to experience vomiting compared to an individual who probably just lost his livelihood. A woman who is pregnant may experience nausea compared to a heavily weighted woman who may experience high blood pressure.

\section{Evaluation of Ride Comfort}

In order to make an effective evaluation of the current metro ride comfort, it is important to understand the sort of forces or accelerations experienced by passengers. When an individual sits in a train, the individual is subjected to several forces due to reactions by the movement of the train. For example, when a train brakes, the passenger feels a pulling force. When the train accelerates, the passengers react as though a force pushes him or her unto the seats. These reactive forces experienced acts in a direction of six degrees of freedom. Each action of forces on the train car body results in a corresponding reaction on the passenger in mostly three out of the six degrees of freedom. As a train commutes from one point to the next, vibrations can be felt by passengers, especially when the vehicle suspension system has a high value of transmissibility.

\subsection{Ride Comfort}

Ride comfort in a train vehicle is mostly evaluated using the mean comfort standard scale method or ISO 2631:1997 
Table 2 Common symptoms caused by motion sickness [3]

\begin{tabular}{llll}
\hline Gastro-related & Somatic & Objective & Emotional \\
\hline Stomach Awareness & Dizziness & Skin humidity & Anxiety \\
Nausea & Exhaustion & Pulse rate & Nervousness \\
Sickness & Increased salivation & Blood Pressure & Scared \\
Retching & Drowsiness & Respiration rate & Apathy \\
Vomiting & Cold sweating & High body temperature & Tense \\
\hline
\end{tabular}

or the Sperling ride index (Werzungzahl) methods [23]. When these methods are considered as not being sufficiently accurate, the running r.m.s. method or fourth power vibration methods are used [23]. These different methods use measured r.m.s. weighted acceleration values got through the use of sensors or accelerometers. The weighted accelerations is defined by the equation below; however, these values were measured for three degrees of freedom, namely

- Vertical vibration

- Longitudinal vibration

- Lateral vibration

\subsection{ISO 2631:1997}

According to ISO-2631, the basic evaluation method uses frequency weighted r.m.s. accelerations defined by the equation below [23]

$a_{\mathrm{w}}=\left[\frac{1}{T} \int_{0}^{T} a_{\mathrm{w}}^{2}(t) \mathrm{d} t\right]^{\frac{1}{2}}$,

where $a_{\mathrm{w}}$ is the r.m.s. acceleration values (as a function of time) and $T$ is the time in seconds.

Likely reactions to various magnitudes of overall vibrations in a public transport vehicle are shown in Table 3.

From Table 3, we see that when the overall vibration in the car body is below $0.3 \mathrm{~m} / \mathrm{s}^{2}$, it is considered that the overall perception of ride comfort of the passenger is that the ride is comfortable. This means that any recorded value above $0.3 \mathrm{~m} / \mathrm{s}^{2}$ would be uncomfortable for the passengers ranging from being slightly uncomfortable to being extremely uncomfortable.

\subsection{Sperling Ride Index (Wz)}

This method is commonly called the Werzungzahl (Wz) method which is a frequency weighted r.m.s. value of accelerations evaluated over given time intervals or over a defined track section [23]. The $\mathrm{Wz}$ formula is given below as
Table 3 R.M.S vibration levels and passenger perception [24]

\begin{tabular}{ll}
\hline R.M.S. vibration level & Passenger perception \\
\hline$<0.315 \mathrm{~m} / \mathrm{s}^{2}$ & Not comfortable \\
$0.315-0.63 \mathrm{~m} / \mathrm{s}^{2}$ & A little uncomfortable \\
$0.5-1 \mathrm{~m} / \mathrm{s}^{2}$ & Fairly uncomfortable \\
$0.8-1.6 \mathrm{~m} / \mathrm{s}^{2}$ & Uncomfortable \\
$1.25-2.5 \mathrm{~m} / \mathrm{s}^{2}$ & Very uncomfortable \\
$>2 \mathrm{~m} / \mathrm{s}^{2}$ & Extremely uncomfortable \\
\hline
\end{tabular}

Table 4 Ride index (Wz) and passenger perception [23]

\begin{tabular}{ll}
\hline Ride index Wz & Vibration sensitivity \\
\hline 1 & Just noticeable \\
2 & Clearly noticeable \\
2.5 & More pronounced but not unpleasant \\
3 & Strong, irregular, but still tolerable \\
3.25 & Very irregular \\
3.5 & Extremely irregular, unpleasant \\
4 & Extremely unpleasant: prolonged exposure harmful \\
\hline
\end{tabular}

$\mathrm{Wz}=4.41\left(a^{\mathrm{wrms}}\right)^{0.3}$

where $a^{\text {wrms }}$ is the r.m.s. value of frequency weighted acceleration $a_{\mathrm{w}}$ in $\mathrm{m} / \mathrm{s}^{2}$.

With the Sperling ride index, each direction has to be determined separately. This makes it less accurate compared to ISO-2631 and also more disadvantageous. Howbeit, it does make for a much simpler read and understanding as its final values are whole numbers. The use of Sperling ride index is most handy when comparing two or more situations. From Table 4, it is seen that with ride index of less than 2.5, the comfort level ranges from being very comfortable to being at a not so pleasant level. Between 2.5 and 3.5, the comfort level ranges from being slightly uncomfortable but tolerable to being very uncomfortable. Beyond this point, passengers become increasingly frustrated, nauseated and they cannot wait for the 
journey to be over. Any higher than 3.5, prolonged exposure becomes harmful and the train could be considered as a hazardous environment.

\subsection{Mean Comfort Standard Method $N_{M V}$}

There is a third method for quantifying customer comfort called the mean comfort standard method [25]. This method, similar to the ISO 2631 method uses values of acceleration from the three translational directions converted into a comfort evaluation scale [26]. This method is not considered as a very reliable and accurate evaluation method due to several reasons including

$$
N_{\mathrm{MV}}=6 \times \sqrt{\left(a_{\mathrm{XP} 95}^{W_{\mathrm{d}}}\right)^{2}+\left(a_{\mathrm{YP} 95}^{W_{\mathrm{d}}}\right)^{2}+\left(a_{\mathrm{ZP} 95}^{W_{\mathrm{d}}}\right)^{2}}
$$

- It is only valid for straight lines and cannot be used in curves.

- It only uses the 95th percentile of frequency weighted acceleration values.

- Final values cannot be correlated to track conditions as critical lateral and vertical values may be kilometres apart.

- There is loss of information as it does not utilise all data values.

As seen in Table 5, the mean comfort standard scale seems quite similar with the Sperling ride index scale. Mean comfort values less than 1.5 are considered to be very comfortable with values between 1.5 and 4.5 ranging from being comfortable to being uncomfortable. Any high than 4.5 , the ride comfort becomes very uncomfortable.

Table 5 Mean comfort standard scale [26]

\begin{tabular}{ll}
\hline$N_{\mathrm{MV}}<1.5$ & Very comfortable \\
\hline $1.5 \leq N_{\mathrm{MV}} \geq 2.5$ & Comfortable \\
$2.5 \leq N_{\mathrm{MV}} \geq 3.5$ & Medium \\
$3.5 \leq N_{\mathrm{MV}} \geq 4.5$ & Uncomfortable \\
$N_{\mathrm{MV}} \geq 4.5$ & Very uncomfortable \\
\hline
\end{tabular}

\subsection{Vibrations}

The vibration on passengers in the metro was measured using "Vibration Monitoring". Vibration Monitoring is a simple app in android app stores which uses accelerometers in a smart phone and measures weighted acceleration changes about a given point of rest of the phone.

\subsubsection{Test Setup}

The vibration data were taken over the following five chosen stations on the metro network shown in Fig. 9:

- Pelaw

- Fellgate

- Brockley Whins

- East Boldon

- Seaburn

Looking specifically at customer comfort, these stations were chosen because they sit in a section where the current metro car reaches its maximum speed of $80 \mathrm{~km} / \mathrm{h}$. Being able to measure the sort of vibrations experienced by passengers as the metro starts from rest to maximum speed, this section of the track proved to be the best choice for the evaluation. The journey was taken starting from Seaburn to Pelaw and back during which speed and vibration data were taken.

The simple test was set up when the phone is placed on a passenger's body (thighs or strapped to the chest) to measure changes in body motion due to vibration experienced. As the train moves on the tracks, every sudden change in direction of the car body results in a corresponding change in the direction of the passenger's body which is recorded by the software. At the same time, speed view uses the phone's internal GPS system, connects to as many satellites as cell reception would allow and records changes in speed of the train as shown in Fig. 10. This process is done in the background. Figure 11 shows the relatively easy setup of vibration testing. As previously explained, vibration monitoring is used as the vibration software, most especially, because of its ability to collect recorded acceleration data from the accelerometers in the phone and this data can be read using Microsoft excel. This
Fig. 9 Analysed section of the metro network

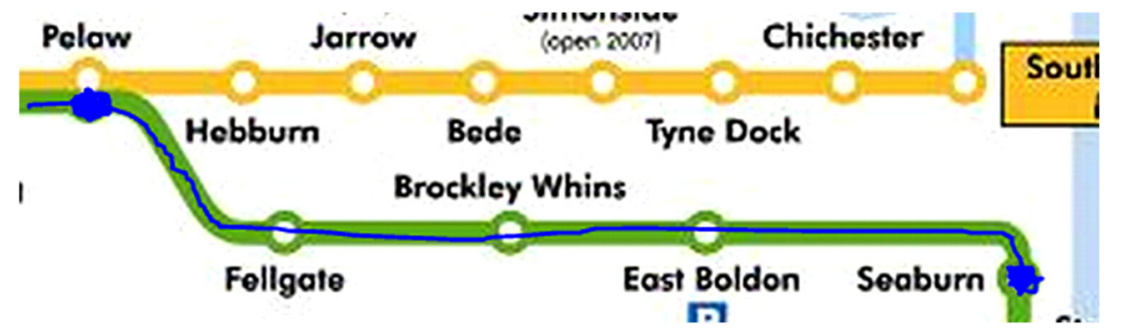


Fig. 10 Speedview setup
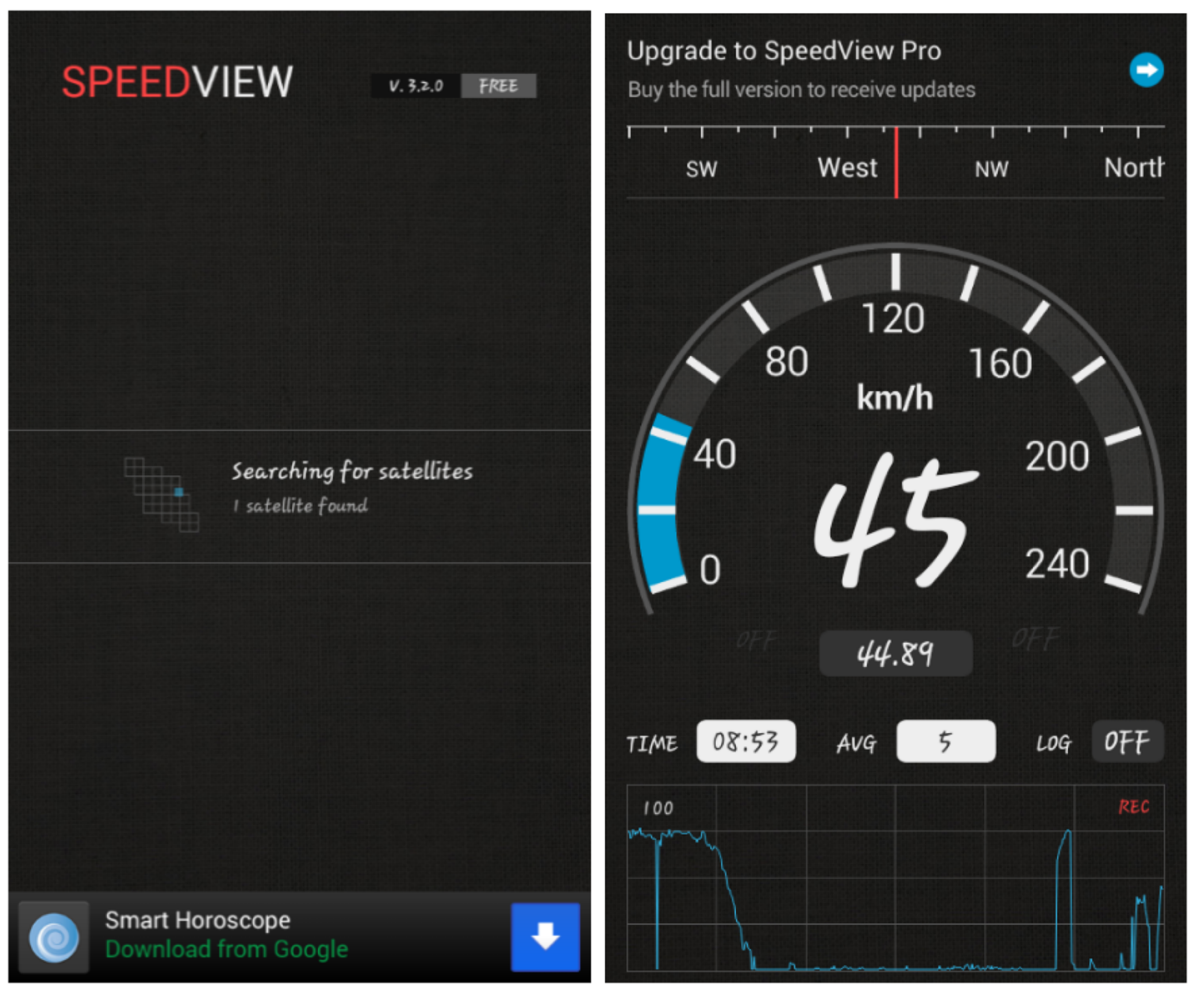

Fig. 11 Vibration monitoring setup

\section{Accelerometer Sensor}

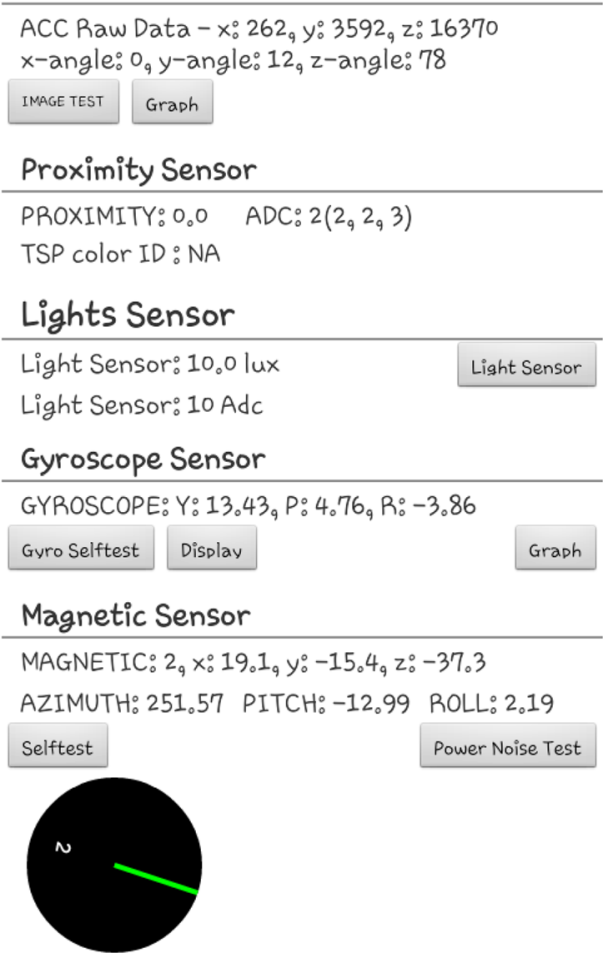

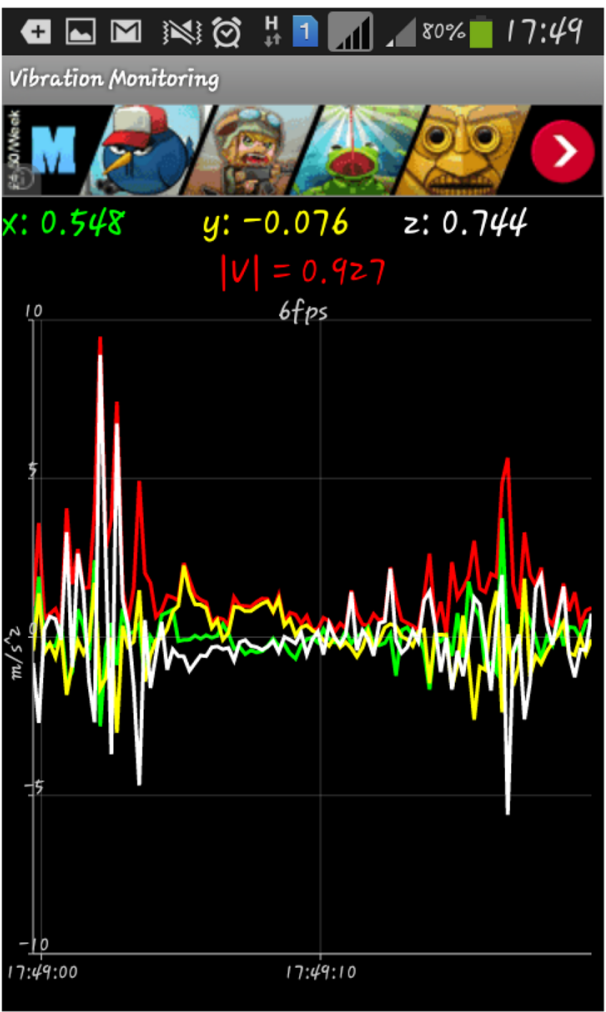

process was better favourable as it saves the need for procurement of accelerometers. As seen in Fig. 11, the measurements are made for all three tri-axial directions and values are shown in a graphical format in real time. $X$, $Y$ and $Z$ directions (lateral, longitudinal and vertical directions, respectively) are indicated by the green, yellow 
and white lines respectively, whilst the red line indicates overall vibration.

\subsubsection{Nature of Data}

The graph is plotted as acceleration against time with acceleration values rising and falling in conjunction with recorded vibration. The green line indicates the comfort threshold below which acceleration values would essentially be comfortable for passengers, whilst the red line is the point above which any acceleration value would be extremely uncomfortable. These lines are based on ISO2631 which puts its scale at 0.3 for comfort and around 0.8 for extreme disturbances in comfort. The zone between 0.3 and 0.8 (green and red lines) ranges from being slightly uncomfortable to being extremely uncomfortable.

\subsubsection{Seaburn to Pelaw}

Figure 12 shows the speed/time performance graph of the metro from seaburn to pelaw. As expected, recorded values show that the metro reaches its maximum speed of $80 \mathrm{~km} / \mathrm{h}$ in all four track sections. The graph also shows some quick changes in speed which is seen as the train accelerates from rest and maintains a relatively constant speed for a specific time frame after which it decelerates to a stop at the next station. A critical analysis of the graph shows that there are also many slight and sudden changes in speed as the vehicle moves along. This is especially seen in the journey from Seaburn to East Boldon and between Fellgate and Pelaw. This is caused by a combination of a not so smooth speed change system in the driver's cabin, poor track conditions and vibrations of the car body which causes slight and sudden losses in momentum. Further analysis of the graph shows that the commuting time between stations follows the advised timetable with the train taking approximately $3 \mathrm{~min}$ from Seaburn to pelaw, $3 \mathrm{~min}$ from East Boldon to Brockley Whins, 2 min from Brockley Whins to Fellgate and finally 6 min from Fellgate to Pelaw.

From Fig. 13, it is seen that from the start of each journey, the vibration level starts well below the comfort line at the stations. Acceleration levels at the stations are between 0.03 and $0.1 \mathrm{~m} / \mathrm{s}^{2}$ and these values were expected although the car body is at rest as there are vibrations due to the car engine and also natural whole vibrating bodies. The amount of vibration increases as the train begins to move away from the station; in the journey between each station, a large amount of vibrations falls between the green and red lines. This indicates that when ever the train is at rest, passengers would feel very minuted vibrations which would be comfortable. However when the train leaves the station, vibrations increase and passengers may feel uncomfortable. Looking at these values in the Sperling ride index, we see similar results with the majority of values falling between the comfortable and extremely uncomfortable lines. A point worth of note is that with the Sperling ride index, $35 \%$ of $\mathrm{Wz}$ values fall well above the red line, indicating that in $35 \%$ of the time, passengers
Fig. 12 Speed/time graph of the metro from Seaburn to Pelaw

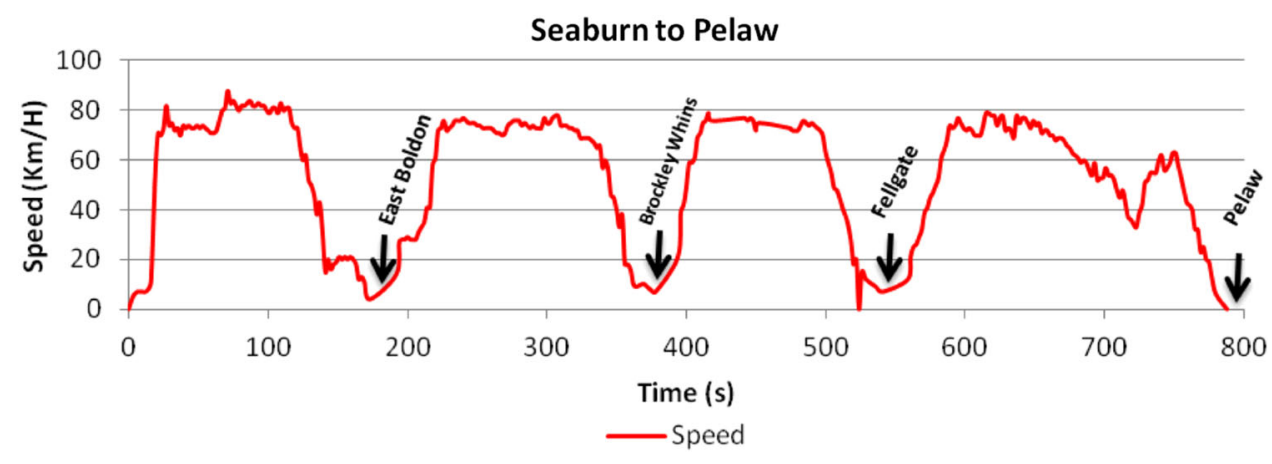

Fig. 13 Seaburn-Pelaw vibration spectrum (time domain)

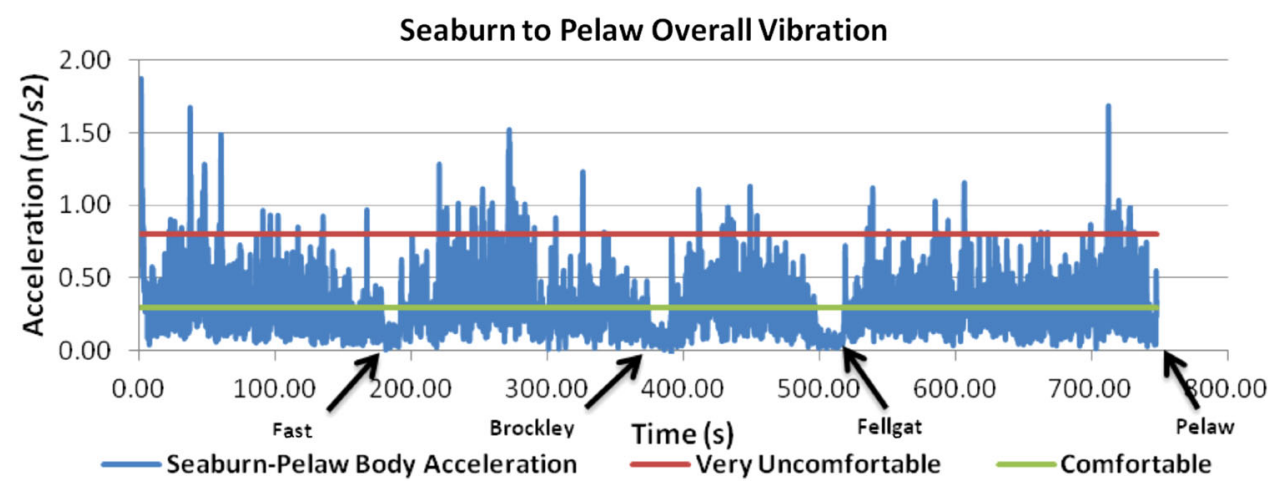



Sperling ride index spectrum
Fig. 14 Seaburn-Pelaw

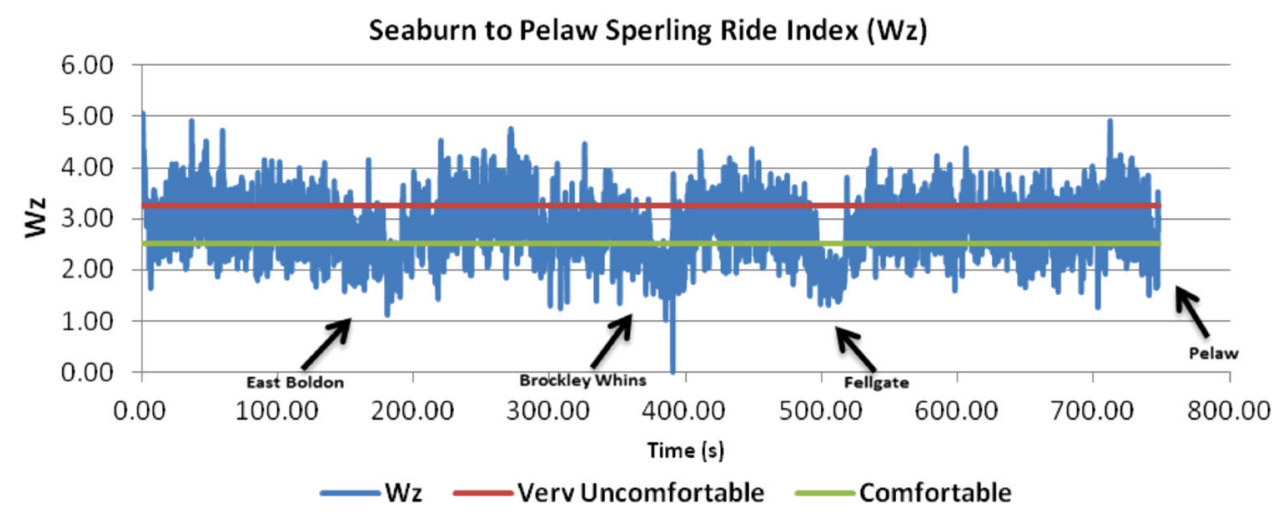

Fig. 15 Pelaw-Seaburn speed graph

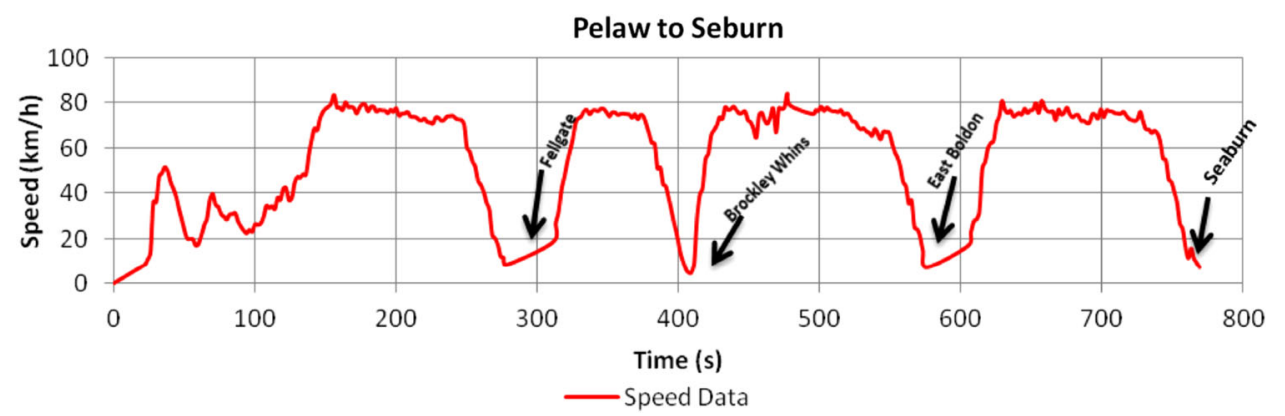

experience very irregular vibrations however it is notable that the Sperling ride index is not as accurate as ISO-2631 [23].

In conclusion, Figs. 13 and 14 indicate that passengers who spent $70 \%$ of their time on the metro will feel vibrations ranging from being slightly uncomfortable to being very uncomfortable. Relating these findings to real situations, it can be predicted that for the current metro system, there may not be extreme cases of vibrations which could make passengers make complaints but there are certainly high magnitude vibrations which would make passengers feel uncomfortable.

\subsubsection{Pelaw to Seaburn}

On the return journey, a different metro car was used in which comparisons can be made on the values of speed and vibrations from different metro cars. It is expected that considering there are 90 cars among the metro fleet, no two car vibrations would be exactly the same. The dip in speed seen in the beginning of the graph in Fig. 15 between Pelaw and Fellgate is due to a combination of a gradient and curve where the train slows down to about $25 \mathrm{~km} / \mathrm{h}$ in order to successfully negotiate the curve whilst climbing the gradient. At this speed, vibration stays normal because the speed is relatively low. Despite the change in the metro car, a smooth speed graph could still not be observed as the same sudden changes in speed are seen all through the line.
This goes to show that even with different metro cars, speed is still affected by poor tracks. Further analysis of Fig. 16 shows that the timings were relatively the same with Fig. 12. This observation supports claims of the metro's high reliability.

The trend of acceleration values is very similar to previous results with over $70 \%$ of recorded values above the comfort threshold line. Notice the increase in vibrations between Brockley Whins and East Boldon. This section of the tracks is generally made up of straight line tracks, where better comfort would be expected; however, there is a definite increase in vibration due to below par suspension systems and poor track conditions. When the overall vibration and speed graphs are overlapped, it is noticed that the train vehicle is travelling at a maximum speed but the speed changes suddenly at the same time. This goes to show how the speed is affected by the vibrations of the car body and vice versa. Figure 17 (Sperling ride index) shows similar results with Fig. 14. The train starts from rest, accelerates to about $80 \mathrm{~km} / \mathrm{h}$ during which point the vibration values rise above the comfort line. Accelerating values fall well below the line as the train decelerates and comes to a stop at each station.

\subsubsection{Conclusion on Comfort Test Results}

After the analysis of test results, it is clearly seen that the current metro system cannot be considered as a 
Fig. 16 Pelaw-Seaburn overall vibration spectrum (time domain)
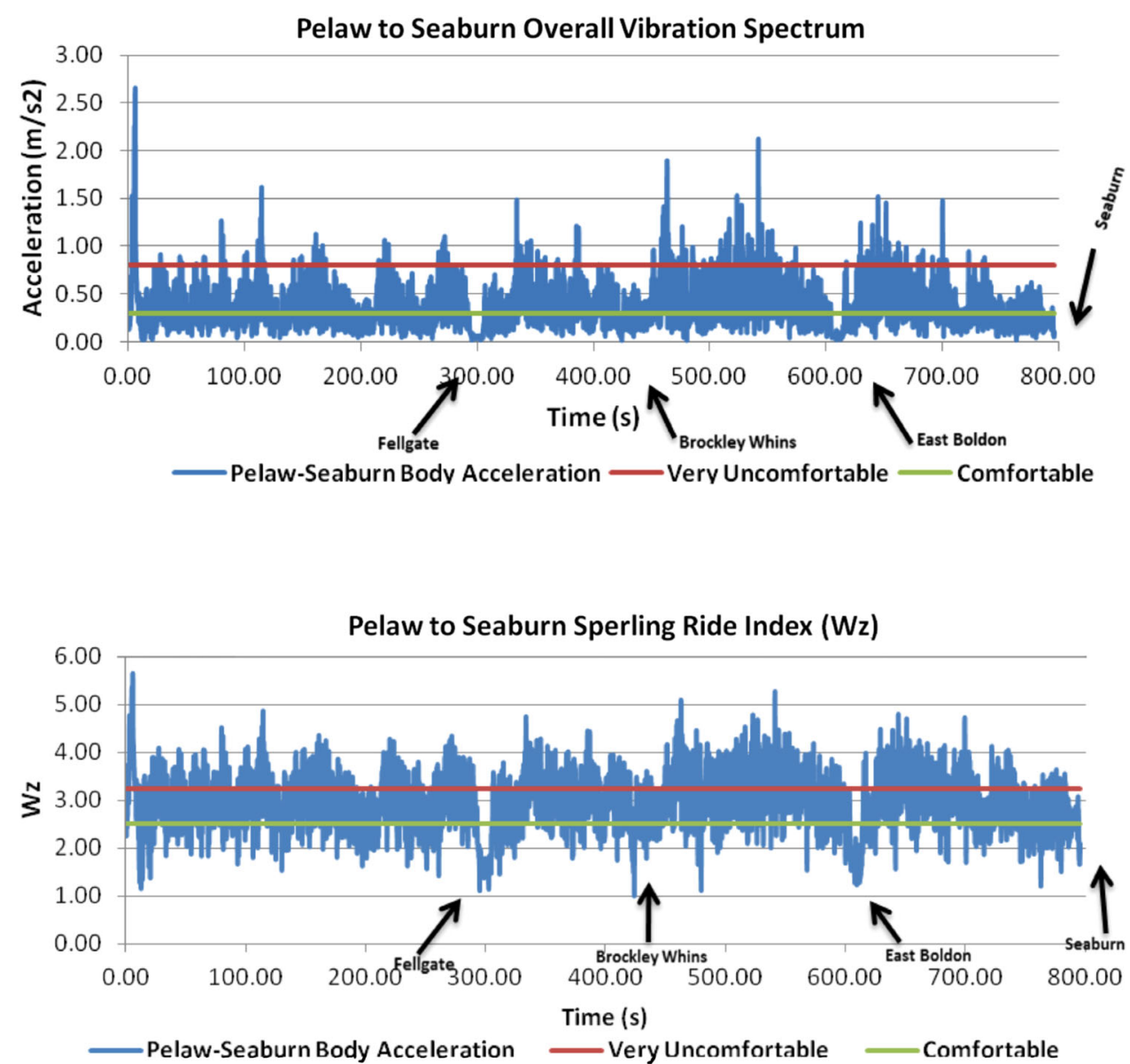

Fig. 17 Pelaw-Seaburn Sperling ride index produce an object-oriented modelling system for railways which could be used to find practical economic solutions to complex railway technology problems [27]. The software is now used as an established railway simulation tool in the railway industries, consultancies and universities in many different countries [27]. OpenTrack allows users the capability to model, simulate, analyse and make decision in various subject areas including

- High-speed rail systems

- Heavy rail

- Intercity rail

- Commuter rail systems

- Heavy haul freight systems

- Mining rail systems

- Metro/subway or underground systems

- People mover systems

- Rack railways and mountain railways

- Maglev systems

The following types of tasks can be achieved using OpenTrack:

- Viewing the capacity of lines and stations

- Rolling stock studies (future requirements) 
- Understanding the requirements for a rail networks infrastructure

- Running time calculation

- Timetable construction

- Evaluation and designing of various signalling systems

- Analysing the effects of system failures

\subsection{OpenTrack Data}

OpenTrack is a simulation software which uses a combination of various data inputs from users and also data already stored in its database to come up with simulation data [27]. User data would normally include but are not limited to

- Tractive effort values

- Rolling resistance values

- Load and weight of the train

- Length of train

- Length of the tracks

- Nature of tracks (for example bad or good adhesion)

- Power

The trains being simulated run according to the timetable on the selected railway network. During simulation, train movements are calculated, and shown in an animated format under constraints imposed by the user through signalling systems and timetable data input. After the simulation, users can display resulting data and analyse these data in the form on diagrams, train graphs, occupation diagrams and statistics.

\subsection{Network Setup}

The first step to running the simulation on OpenTrack is by first building the network to be simulated [28]. Having already chosen five initial stations (Fig. 18), building the network simply requires using the build line tool to build lines connecting each designated station and setting a specific distance between each of them.

Determining distances between each station was obtained using Google maps as shown in Fig. 19 and values were subsequently logged into OpenTrack database. Values observed from the Google maps are as follows:

- Seaburn-Brockley Whins $=2.9 \mathrm{~km}$ or $2900 \mathrm{~m}$

- Brockley Whins-East Boldon $=2.7 \mathrm{~km}$ or $2700 \mathrm{~m}$

- East Boldon-Fellgate $=1.8 \mathrm{~km}$ or $1800 \mathrm{~m}$

- Fellgate-Pelaw $=3.7 \mathrm{~km}$ or $3700 \mathrm{~m}$

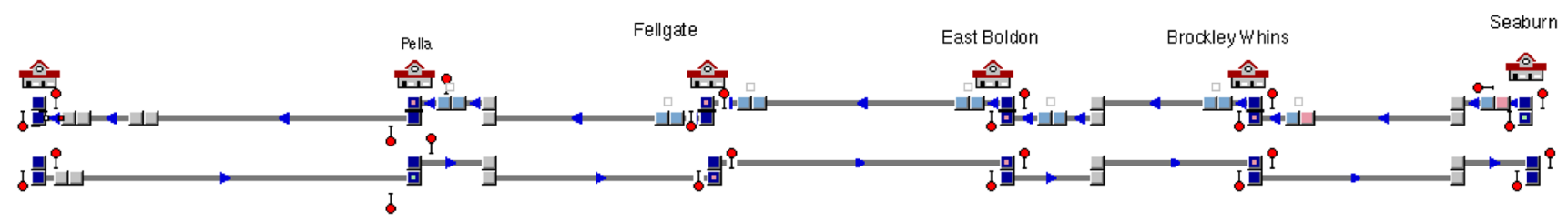

Fig. 18 Built network line on OpenTrack

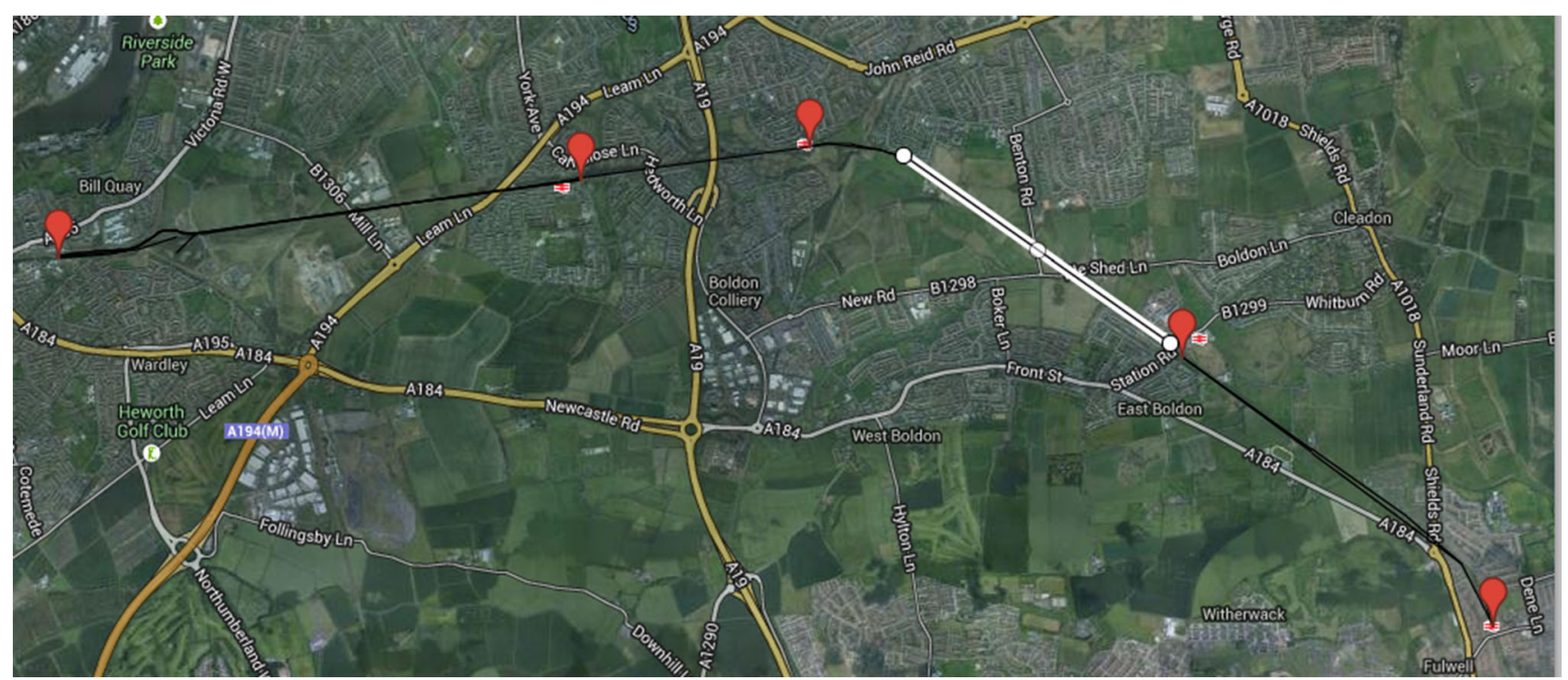

Fig. 19 Google map indications of station location [29] 
Unfortunately, all track gradients and curve radius could not be accurately ascertained from available tools or materials thus these values were roughly estimated and put into the database.

\subsection{Engine Setup}

Input of engine characteristics was done using the engine tool. OpenTrack allows the user to set maximum speed limit by simply defining the necessary tractive effort graph which automatically allocates necessary parameters into the tool [28]. Weight and adhesion values of a fully loaded metro fleet are known as 39 tonnes and length of the train is also known as $27 \mathrm{~m}$. Input of these values allows replication of the current metro system; thus, any change to these values can be easily noticed and compared.

\subsection{Train Setup}

After setup of the engine is completed, the next step is making sure the engine runs as a train. The train tool helps us achieve this through a selection of trains already in the database or if none are found to be useful [28], the user can create a new train and attach engine parameters to it. Two simulations were made. The first being a replication of the current metro system, whilst the second is the replication of a tilting train. The train type chosen was a commuter train with a deceleration rate of $0.68 \mathrm{~m} / \mathrm{s}^{2}$. This was used because it comes closest to the current metro system. Engine parameters are subsequently copied unto the train tool thus making the setup complete.

\subsection{Timetable Setup}

During the simulation, the train tries to obey an already set timetable. Timetable data have already been analysed from the current metro timetables and also measured time taken between stations. Approximate time between each stations is given below:

- Seaburn-Brockley Whins = $3 \mathrm{~min}$

- Brockley Whins-East Boldon = $3 \mathrm{~min}$

- East Boldon-Fellgate $=2 \mathrm{~min}$

- Fellgate-Pelaw $=5 \mathrm{~min}$
These values were put into the system's timetable tool whilst strict checkbox is unchecked. This allows the simulation to run correctly whilst not necessarily adhering to the input timetable. This setting allows the viewing of any differences between real situations and ideal situations. In a real situation, extra times are added to actual times to allow for possible delays of unforeseen circumstances. This simulation would show the metro car behaviour in an ideal situation and total time taken between trains. Dwelling time value of $30 \mathrm{~s}$ is used to allocate the required amount of time the system would take to stop at each station. This would replicate the metro train stopping and starting at each station which allows passengers to embark or disembark from the vehicle.

\subsection{Simulation}

After all required routes, paths, parameters and system characteristics have been built, simulation is started. The simulation tool is used which shows the progress of the train as it commutes from each station to the next. Two simulations were made using this system with the first aimed at replication of the present situation of the metro and the second done with slight change of system parameter in order to simulate a tilting system as closely as possible. The simulation runs from Seaburn station to Pelaw station on the following assumptions:

- It is a clear sunny day, thus adhesion between track and wheel is good.

- There are no tunnels between all five stations thus tunnel adhesion is good.

- The train vehicle is in impeccable condition thus performance is $100 \%$.

- Track quality is good.

\subsubsection{Metro Simulation}

Figure 20 shows how OpenTrack simulates the train. The simulation tool is used and at the click of the start button, simulation is started. A green label is seen moving on the built network indicating that the built network is valid and trains would be able to commute. Time stamps show the progress being made by the train as it accelerates and

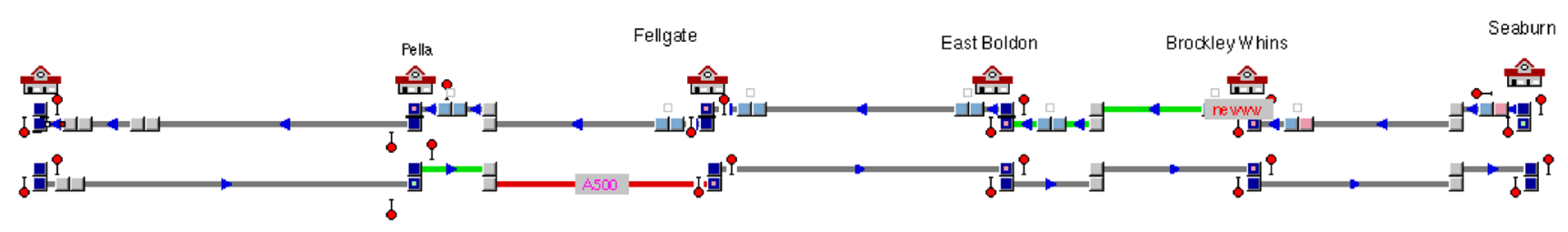

Fig. 20 Simulation of metro 
decelerates at each station. With this simulation, the train takes approximately $11 \mathrm{~min}$ and $44 \mathrm{~s}$ (706 $\mathrm{min}$ ) to move from Seaburn to Pelaw. The real situation shown in Fig. 13 takes approximately $13+\min$. Slight difference can be explained by the dwelling time of the train at each station which can vary widely depending if train drivers wait for longer or shorter times for passengers in cases where the train arrives earlier or later than scheduled time. Approximate dwelling time for this train simulation was set at $30 \mathrm{~s}$ for each station.

\subsubsection{Tilting Simulation}

Several parameters like performance and timetable constrains were changed in the system so as to closely mimic tilting technology systems. Scientific research claims that tilting technology reduces commuting time by as much as $15 \%$ [12]; however, since the current metro actually reaches its maximum speed of $80 \mathrm{~km} / \mathrm{h}$, a new metro engine has to be incorporated and this is done in this simulation by increasing engine parameters by approximately $15 \%$. After simulation, it was observed that the train takes approximately $9 \mathrm{~min} 48 \mathrm{~s}$ to make the journey. When compared with the previous simulation, it is found that a $17 \%$ reduction in commuting time was achieved. If such is the case, it can be claimed that a $17 \%$ increase in engine power leads to a $17 \%$ decrease in commuting time. This information would come in handy in managerial decisions which would need to be made in the near future.

\subsection{Results}

Results achieved confirmed the notion that tilting technology can reduce commuting time in any given journey. Both simulations gave an output similar to real data and this is evident when they are plotted on the same graph. In Fig. 21, it is seen that when compared to real situations, travel time is reduced by as much as $10 \%$ with a $15 \%$ increase in maximum achievable speed. It is noted that the time difference between the real and non-tilt simulated situations is about $40-50 \mathrm{~s}$. This can be explained by differences in dwelling times in both situations. The differences of the sum of each station dwelling time would give the difference in time shown on the graph. For example, if in real situations, average dwelling time for each station is $40 \mathrm{~s}$, the sum would be $2 \mathrm{~min}$. The average dwelling time chosen for simulation is $30 \mathrm{~s}$ with total dwelling time summed up as $1 \mathrm{~min}$ and $30 \mathrm{~s}$. Difference between both dwelling times gives the lag time between real and simulated time values. Other results observed include

- Sudden changes in speed due to poor track conditions and vibrations are virtually eliminated or non-existent as the state of poor track conditions were set as good.

- The dip in speed normally seen between Fellgate and Pelaw is still seen; however, the simulated trains seem to be able to negotiate this curve at a much higher speed. The tilting train simulation travels at $55 \mathrm{~km} / \mathrm{h}$ at this point whilst the non-tilting train travels at $40 \mathrm{~km} / \mathrm{h}$. That means the non-tilting train travels $27 \%$ slower than the tilting train at this curve.

- The maximum speed of the tilting train as expected is $90+\mathrm{km} / \mathrm{h}$ and it maintains this speed for about $1 \mathrm{~min}$. An interesting observation is seen with the old metro which maintains its maximum seep of $80 \mathrm{~km} / \mathrm{h}$ for almost $2 \mathrm{~min}$. It is expected that the new metro would be faster at this section but it is unexpected that it would be almost $50 \%$ faster. A future research could be made to ascertain why this is.

\subsubsection{Accuracy and Validation}

Both vibration and speed analysis done on the metro were done in a manner which could produce as accurate a result as possible; however, several parameters could reduce or undermine the maximum accuracy of the tests. Possible problems which could undermine the maximum achievable accuracy could include

- A strapped phone to the thighs or chest may have some inaccurate readings arising from normal human body
Fig. 21 Speed comparisons between real and simulated speed graphs

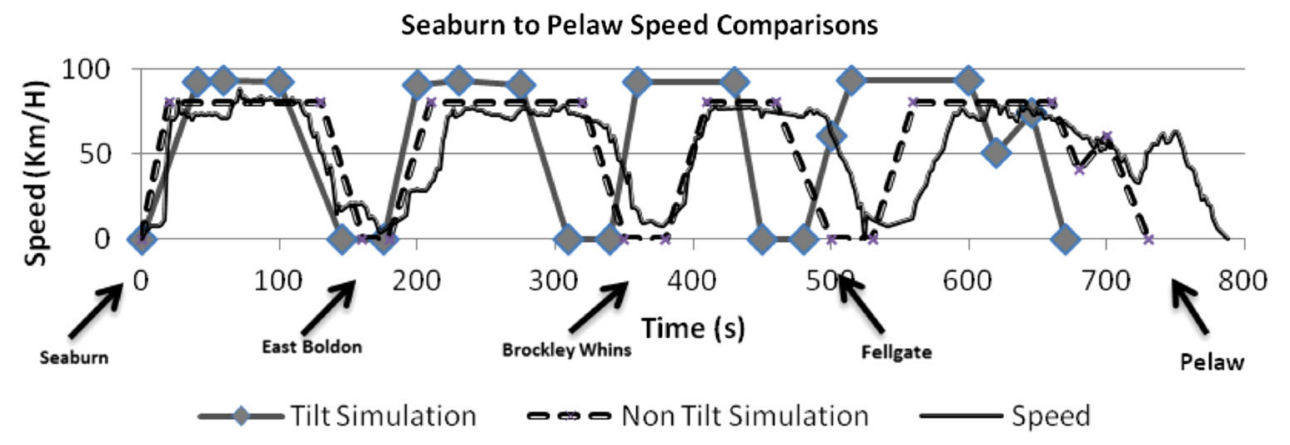


movement (movements not induced by train motion) although conscious efforts were made to limit this factor as much as possible. It can be stated with $100 \%$ confidence that at least $95 \%$ of data recorded in all vibration tests were due to train movements.

- Phone app data may not be as accurate as accelerometers, however it can be predicted with confidence that actually accelerometer values would be slightly higher than phone app data values.

- The tests only confirmed that the current metro system does require an increase in ride comfort, it does not determine the effects of tilting technology. Unfortunately, accurately determining tilting technology effects on the metro cannot be done without a properly modelled system either with prototype train assemblies or a simple similar test done on a working tilting train. All options are limited by time allocated for this project.

- Using a phone app as opposed to actual accelerometer devices which could give better readings of vibration.

- Speed graphs were done also using a phone app which may or may not have been affected by cell reception at the time. However during testing, it can be stated with reasonable confidence that this is not the case.

- Replication of the Tyne and Wear Metro network was not $100 \%$ complete due to insufficient curve data of the network although every endeavour was made to input major curve data on the network.

All of these factors may reduce the overall level of accuracy of these tests but it can also be confidently stated that the tests were successful in reasonably identifying the level of comfort currently experienced by the passengers and the level reduced travel time should tilting technology be incorporated in the current metro fleet.

\section{Conclusion and Discussion}

A review of test results shows that tilting technology does have the merits of being a suitable choice of technology which could improve both performance, commuting time and ride comfort. This project has shown that in the current metro network

- An increase to a top speed of $93 \mathrm{~km} / \mathrm{h}$ is achievable on the metro network through the use of tilting technology.

- The current metro system may not be overly uncomfortable but could do with significant improvements to ride comfort which can be provided by tilting technology.

- Commuting time can be increased by as much as $10 \%$ for every $15 \%$ increase in speed which can be provided by tilting technology.
- With the current metro fleet scoring high in majority of customer satisfaction factors, an increase in comfort would significantly increase passenger patronization.

In conclusion, from a management point of view, these benefits may not be enough to properly pursue implementation of tilting technology in the current metro system. A cost/benefit analysis would likely be the deciding factor should such a decision be made. Considerations on the amount of infrastructural upgrading of each train fleet or whole track network makes for a much less optimistic view of tilting technology systems being used in increasing customer satisfaction, speed or even customer comfort. With the current metro system really only in need of a comfort upgrade, the amount of upgrades required to implement tilting technology just does not in the author's opinion seem like a wise business decision.

\subsection{Recommendations}

A more reasonable solution could be the implementation of a system which does not necessarily improve speed but definitely improves ride comfort without the vast need for system upgrades. Obviously such a system would be mainly focused on the vehicle suspension. One such system being researched with such promise of improving ride comfort is the active or semi-active lateral suspension (ALS) system researched by FIAT [30]. This is a system which basically works by introducing fast controlled hydraulic dampers placed both vertically and longitudinally on the bogie frame. The system can also be combined with tilting technology systems and has been claimed to have the capabilities of increasing comfort by over $37 \%$ [30, p. 10]. Figure 22 shows the possible impacts of a $37 \%$ decrease in overall vibrations of the current metro system and it immediately becomes apparent that the impacts could be quite positive. The level of comfort falls well

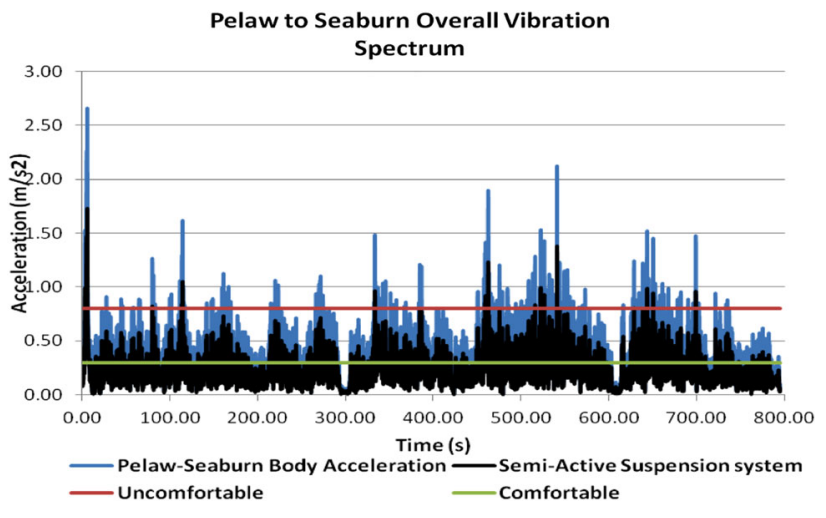

Fig. 22 Data showing possible impacts of implemented active suspension systems on metro 
below the red line with more than $95 \%$ of acceleration values below this line. Considering the availability of such a system, it may be more logical or economic to implement an active suspension system as opposed to a tilting technology system.

\subsection{Future Study}

Future studies on this topic should focus on ALS systems and its suitability to ride comfort. If the current metro system is to be improved, emphasis should be placed on ride comfort. As this study has confirmed that tilting technology may be beneficial to improving speed and comfort, it has noted that expenditures required for such an upgrade far surpasses the required benefits. ALS systems have been proven to work impeccably well in the automotive industry [31, 32] and is being fully researched for train applications [33-35]. It can be predicted that considering speed is not important in the current metro network, an implementation of active suspension systems can meet the required comfort levels which would warrant highly increased customer satisfaction without the need for an "over the top" overhaul of the entire metro fleet and network. Happy customers make for increased revenues and considering that the current metro scores highly in many customer satisfaction factors, growth appears to be limited by its current level of ride comfort.

Open Access This article is distributed under the terms of the Creative Commons Attribution 4.0 International License (http:// creativecommons.org/licenses/by/4.0/), which permits unrestricted use, distribution, and reproduction in any medium, provided you give appropriate credit to the original author(s) and the source, provide a link to the Creative Commons license, and indicate if changes were made.

\section{References}

1. Stout KL (2013) Deadly high speed train crash. CNN, Hong Kong

2. Kim NP, Park TW (1999) A study on the dynamic performance of the $200 \mathrm{~km} / \mathrm{h}$ Korean tilting train by means of roller rig test. J Mech Sci Technol 23:910-913

3. Persson R (2011) Tilting trains: enhanced benefits and strategies for less motion sickness. KTH Royal Institute of Technology, Stockholm

4. Forstberg J, Andersson E, Ledin T (1988) Influence of different conditions for tilt compensation on symptoms of motion sickness in tilting trains. Brain Res Bull 47(5):525-535

5. Barnett R (1992) Tilting trains: the Italian ETR and the Swedish X-2000, vol 113. California High Speed Rail Series, California

6. Yashushi N, Yoshi S, Shoji N, Hiroaki N, Toshiaki H (1997) Tilting control system for railway vehicle using long-stroke air springs

7. Shikimura A, Inaba T, Kakinuma H, Sato I, Sato Y, Sasaki K, Hirayama M Development of Next-generation Tilting Train by Hybrid Tilt System. s.l.: Hokkaido Railway Company, Sapporo; Railway Technical Research Institute, Kokubunji
8. Zhou R, Zolotas A, Goodall R (2011) Integrated tilt with active lateral secondary suspension control for high speed railway vehicles. Mechatronics 21:1108-1122

9. Nedall B-L (1998) The experience of the SJ X2000 tilting train and its effect on the market. 103, In: Proceedings of the institution of mechanical engineers. J Rail Rapid Transit Part F 212:103-108.

10. Persson R (2008) Tilting trains: technology, benefits and motion sickness. Stockholm Network, Stockholm

11. Hitachi-rail.com (2014) Pneumatic mechanism. http://www.hita chi-rail.com/products/rolling_stock/tilting/feature04.html. Accessed 17 April 2014

12. Frostberg J (2000) Ride comfort and motion sickness in tilting trains: human responses to motion experiments and simulator experiments (TRITA-FKT). Adtranz, Sweden

13. Pearson JT, Goodall RM, Pratt I (1998) Control system studies of an active anti-roll bar tilt system for railway vehicles. J Rail Rapid Transit 43:212

14. Persson R (2007) Tilting trains: description and analysis of the present situation. KTH Rail Vehicles, Stockholm

15. Hitachirail.com (2013) Predictive tilt control system. http://www. hitachi-rail.com/products/rolling_stock/tilting/feature02.html. Accessed 06 Jan 2014

16. Urbanrail.net (2014) Newcastle-upon-Tyne. http://www.urban rail.net/eu/uk/new/newcstle.htm. Accessed 25 Aug 2014

17. Railway-technology.com (2013) Tyne \& Wear Metro, United Kingdom. http://www.railway-technology.com/projects/tyne/. Accessed 20 July 2014

18. NEXUS (2014) Metro Strategy 2030: background information. http://www.nexus.org.uk/sites/default/files/Metro\%20Strategy\% $202030 \% 20$ summary\%20document.pdf. Accessed 30 April 2014

19. Anna H (2014) Plans to expand the Tyne and wear metro. http:// www.capitalfm.com/northeast/on-air/news-travel/local-news/plans-toexpand-the-tyne-and-wear-metro/. Accessed 28 June 2014

20. Lauriks G, Evan J, Förstberg J, Balli M and Barron de Angoiti I (2003) UIC comfort test. http://www.vti.se/en/publications/pdf/ uic-comfort-tests-investigation-of-ride-comfort-and-comfort-dis turbance-on-transition-and-circular-curves.pdf. Accessed 26 April 2014

21. Greater-Manchester-Commitee (2014) Transport for customer satisfaction survey: rail passenger survey autumn 2013.Transport for greater Manchester committee report for information, Manchester

22. Frostberg J (2000) Ride comfort and motion sickness in tilting trains: human responses to motion environments in train and simulator experiments. s.1.: TRITA-FKT

23. Ramasamy N, Shafiquzzaman K, Mats B,Virendra Kumar G, Huzur Saran V, Harsha SP (2014) Determination of activity comfort in Swedish passenger trains. http://www.uic.org/cdrom/ 2008/11_wcrr2008/pdf/R.2.4.3.2.pdf. Accessed 12 April 2014

24. Sathishkumar P, Jancirani J, Dennie J (2014) Reducing the seat vibration of vehicle by semi active force control technique 2 . J Mech Sci Technol 28:473-479

25. Annelli O (2010) Methods for reducing vertical carbody Vibrations of a rail vehicle. KTH Engineering Sciences, Sweden

26. Kufver, B (2014) EN standard 12299 for evaluation of ride comfort for rail passengers. http://www.railwaygroup.kth.se/polo poly_fs/1.347076!/Menu/general/column-content/attachment/Bj\% C3\%B6rn\%20Kufver\%201.pdf. Accessed 08 Dec 2014

27. OpenTrack.ch (2014). OpenTrack railway simulation. http://www. opentrack.ch/opentrack/opentrack_e/opentrack_e.html. Accessed 08 April 2014

28. Huerlimann D, Nash AB (2014) OpenTrak manual, simulation of railway networks. s.l.: OpenTrack Railway Technology Ltd and ETH Zurich Institute for Transport Planning and Systems

29. Google (2014) Seaburn-Pelaw track map. s.l.: Google maps

30. Montiglio M, Stefanini A (1999) Development of a semi-active lateral suspension for a new tilting train. s.l.: Fiat Research Centre 
31. van der Sandea TPJ, Gysen BLJ, Besselink IJM, Paulides JJH, Monolova EA (2013) Robust control of an electromagnetic active suspension system: simulations and measurements 2. Mechatronics 23:204-212

32. Abduljabbar ZS, ElMadany MM (2000) Optimal active suspension with preview for a quarter-car model incorporating integral constraint and vibration absorber. Cairo-Egypt: international MDP conference
33. Sasaki K (2000) A lateral semi-active suspension of tilting trains. 1, QR of RTRI, vol. 41, Ken-Yusha, Tokyo

34. Foo E, Goodall RM (2000) Active suspension control of flexiblebodied railway vehicles using electro-hydraulic and electromagnetic actuators 5. Control Eng Prac 8:507-518

35. Tanifujia K, Koizumi S, Shimamune R (2002) Mechatronics in Japanese rail vehicles: active and semi-active suspensions 9. Control Eng Prac 10:999-1004 